\title{
Oleanene Glycosides of the Aerial Parts and Seeds of Bupleurum falcatum and the Aerial Parts of Bupleurum rotundifolium, and Their Evaluation as Anti-hepatitis Agents
}

\author{
Yuko Nakahara, ${ }^{a}$ Masafumi Okawa, ${ }^{b}$ Junei Kinjo, ${ }^{b}$ and Toshihiro NoharA ${ }^{*, c}$ \\ ${ }^{a}$ Graduate School of Pharmaceutical Sciences, Faculty of Life Sciences, Kumamoto University; 5-1 Oe-honmachi, \\ Kumamoto 862-0973, Japan: ${ }^{b}$ Faculty of Pharmaceutical Sciences, Fukuoka University; 8-19-1 Nanakuma, Higashi-ku, \\ Fukuoka 814-0180, Japan: and ${ }^{c}$ Faculty of Pharmaceutical Sciences, Sojo University; 4-22-1 Ikeda, Kumamoto \\ 860-0082, Japan. Received May 25, 2011; accepted August 18, 2011; published online August 23, 2011
}

To facilitate effective resource utilization, we have investigated triterpene saponins such as saikosaponin from the aerial parts of Bupleurum (B.) falcatum L., which are commonly discarded. Seven oleanene saponins were isolated from this plant; they were classified as the 13,28-epoxy type, 12-ene type, 9(11),12-diene type, and 28-acid type on the basis of their structural characteristics. For comparison, we also examined the oleanene saponins of the seeds of $B$. falcatum and the aerial parts of $B$. rotundifolium $L$. to obtain seven saponins and one sapogenol from the former and thirteen oleanene saponins from the latter. Several compounds obtained from them were investigated for their hepatoprotective activity and hepatotoxicity. The 13,28-epoxy type saponins had hepatoprotectivity. Ursane type showed hepatotoxicity from middle concentration. The 11,13(18)-diene type saponins did not express hepatoprotective activity. The 28 -acid type saponin which has a glucosyl carboxy group showed hepatoprotective action.

Key words Bupleurum falcatum; saikosaponin; hepatoprotective activity; Bupleurum rotundifolium

Bupleuri Radix appeared in 'Shen hong ben cao jing' as a drug harmless to humans and one of the most important Chinese crude drugs used as an antipyretic and anti-inflammatory. The occurrence of saikosaponins (saikosaponin $\mathrm{a}: \mathrm{c}: \mathrm{d}=2: 1: 3$ ) in this drug and their functions in peptide synthesis and detoxification in the liver are well known. Many earlier works were reported, as follows. Shibata et al. reported isolation of various saponins from Bupleuri Radix originating from Bupleurum falcatum cultivated in Japan and the liver-protecting activity of a mixture of the major saponin, saikosaponin a, with other saponins. ${ }^{1)}$ Shimaoka et $a l$. reinvestigated the structure of saikosaponin, ${ }^{2)}$ and Tori et al. conducted a ${ }^{13} \mathrm{C}-\mathrm{NMR}$ investigation of saikosapogenins. ${ }^{3)}$ Ogihara and colleagues synthesized artificial derivatives of saikosaponins by using an acid or enzyme ${ }^{4,5)}$ and examined their function of accelerating corticosteroid secretion. $\left.{ }^{6}\right)$ Mitsuhashi and colleagues isolated a new saikosaponin malonate from B. scorzonerifolium, ${ }^{7)}$ and Yoshikawa et al. isolated a new saponin from B. scorzonerifolium and studied its liver-protecting components. ${ }^{8,9}$ Moreover, agricultural studies of the constituents of the seeds have been begun by Ono et al. ${ }^{10)}$ and studies of anti-proliferation of tumor cells have been initiated by Mihashi and colleagues. ${ }^{11,12)}$

In some areas in Kumamoto Prefecture, this plant is cultivated, and its aerial parts are discarded. We investigated the drug's constituents in conjunction with studies of the aerial parts of B. rotundifolium, which is cultivated for cut flowers, and the seeds of $B$. falcatum. We also examined the liver-protecting functions of the obtained specimens and elucidated the relationship between their structure and activity.

Harvested aerial parts $(2.6 \mathrm{~kg})$ of $B$. falcatum were extracted by refluxing with $\mathrm{MeOH}$ to give an extract (262.2 g), which was then separated by using the solvent partition method. Various chromatographies on Diaion HP-20, Sephadex LH-20, silica gel, and Chromatorex octadecylsilyl (ODS) silica were applied to provide BFA-5 (108 mg),
BFA-6 (264 mg), BFA-8 (45 mg), BFA-11 (21 mg), BFA-13 (110 mg), BFA-17 (21 mg), BFA-20 (20 mg), BFA-22 (72 mg), BFA-25 (17 mg), BFA-27 (110 mg), BFA-28 (680 mg), and BFA-29 (20 mg).

BFA-8 (1), BFA-22 (2), BFA-5 (3), BFA-6 (4), and BFA25 (5) were identified as saikosaponin $a,{ }^{3)}$ saikosaponin $\mathrm{c},{ }^{3)}$ saikosaponin $\mathrm{f}^{3)}$ saikosaponin $\mathrm{b}_{2}{ }^{2)}$ and 4 "-O-acetylsaikosaponin $b_{2},{ }^{13)}$ respectively, by fast atom bombardment-mass spectrometry (FAB-MS), ${ }^{1} \mathrm{H}$ - and ${ }^{13} \mathrm{C}-\mathrm{NMR}$ spectra, and chemical reactions.

BFA-20 (6) was obtained as an amorphous powder having $[\alpha]_{\mathrm{D}}-11.8^{\circ}(\mathrm{MeOH})$. Positive high resolution (HR)-FABMS revealed a molecular formula of $\mathrm{C}_{48} \mathrm{H}_{78} \mathrm{O}_{18} \mathrm{Na}$ at $\mathrm{m} / \mathrm{z}$ $965.5088[\mathrm{M}+\mathrm{Na}]^{+}$. The ${ }^{1} \mathrm{H}-\mathrm{NMR}$ spectrum displayed signals due to six tertiary methyl groups at $\delta 0.90,0.96,1.00$, $1.00,1.06$, and 1.66 (each $3 \mathrm{H}, \mathrm{s}$ ); one methyl pentosyl methyl group ( $\left.3 \mathrm{H}, \mathrm{d}, J=6.1 \mathrm{~Hz}, \mathrm{H}_{3}-6\right)$ at $\delta 1.66$; two hexosyl anomeric protons at $\delta 4.94$ and 5.01 (each $1 \mathrm{H}$, anomeric proton, d, $J=7.9 \mathrm{~Hz}$ ); one methyl pentosyl anomeric proton at $\delta$ $5.87(1 \mathrm{H}, \mathrm{s})$; and two olefinic protons at $\delta 5.67$ and $6.68(1 \mathrm{H}$, d, $J=11.0 \mathrm{~Hz}$ and $1 \mathrm{H}$, br d, $J=8.5 \mathrm{~Hz}$, respectively). The ${ }^{13} \mathrm{C}-$ NMR spectrum (Table 1) displayed a total of 48 carbon signals, of which 30 were attributable to saikogenin D comprised of BFA-6 (4). The remaining carbon signals arose from sugar moieties and coincided with those of the sugar moieties in BFA-22 (2), as listed in Table 1. Therefore, the structure of $\mathbf{6}$ was characterized as $\beta$-D-glucopyranosyl$(1 \rightarrow 16)$-[ $\alpha$-L-rhamnopyranosyl-( $1 \rightarrow 4)]$ - $\beta$-D-glucopyranosyl $3 \beta, 16 \alpha, 23,28$-tetrahydroxyolean-11,13(18)-diene. ${ }^{4)}$

BFA-17 (7) was obtained as an amorphous powder having $[\alpha]_{\mathrm{D}}+45.9^{\circ}(\mathrm{MeOH})$. Positive HR-FAB-MS revealed a molecular formula of $\mathrm{C}_{36} \mathrm{H}_{58} \mathrm{O}_{9} \mathrm{Na}$ at $\mathrm{m} / z 657.3982[\mathrm{M}+\mathrm{Na}]^{+}$. The ${ }^{1} \mathrm{H}-\mathrm{NMR}$ spectrum displayed signals due to six tertiary methyl groups at $\delta 0.81,0.97,1.10,1.21,1.28$, and 1.35 (each $3 \mathrm{H}, \mathrm{s}$ ); one olefinic proton at $\delta 5.44$; and one hexosyl anomeric proton at $\delta 6.33(1 \mathrm{H}, \mathrm{d}, J=7.9 \mathrm{~Hz})$. Since the ${ }^{13} \mathrm{C}$ - 
Table $1 .{ }^{13} \mathrm{C}$-Data for Compounds $\mathbf{1}-\mathbf{7}$

\begin{tabular}{|c|c|c|c|c|c|c|c|}
\hline & 1 & 2 & 3 & 4 & 5 & 6 & 7 \\
\hline C-1 & 38.6 & 38.4 & 38.8 & 38.4 & 38.4 & 38.3 & 38.6 \\
\hline 2 & 26.0 & 26.4 & 26.5 & 26.1 & 26.1 & 25.9 & 26.3 \\
\hline 3 & 81.6 & 89.0 & 89.0 & 81.7 & 81.7 & 82.1 & 78.9 \\
\hline 4 & 43.7 & 39.6 & 39.5 & 43.7 & 43.7 & 43.6 & 39.4 \\
\hline 5 & 47.3 & 55.2 & 55.7 & 47.3 & 47.4 & 47.3 & 55.7 \\
\hline 6 & 17.2 & 18.4 & 18.5 & 18.8 & 18.3 & 18.3 & 18.5 \\
\hline 7 & 31.5 & 31.8 & 33.4 & 31.9 & 31.9 & 31.9 & 32.4 \\
\hline 8 & 42.2 & 42.1 & 40.1 & 41.1 & 41.9 & 41.1 & 39.9 \\
\hline 9 & 53.0 & 52.8 & 47.0 & 54.0 & 54.0 & 53.9 & 48.0 \\
\hline 10 & 36.2 & 36.3 & 36.7 & 36.5 & 36.5 & 36.3 & 36.0 \\
\hline 11 & 132.2 & 132.1 & 24.1 & 126.2 & 126.3 & 126.2 & 23.7 \\
\hline 12 & 131.1 & 131.1 & 122.6 & 126.2 & 126.2 & 126.2 & 123.0 \\
\hline 13 & 84.0 & 84.0 & 143.9 & 136.0 & 136.0 & 136.1 & 144.0 \\
\hline 14 & 45.6 & 45.6 & 43.8 & 41.8 & 41.1 & 41.9 & 42.2 \\
\hline 15 & 36.1 & 36.0 & 36.7 & 32.3 & 32.3 & 32.3 & 33.1 \\
\hline 16 & 64.0 & 64.0 & 66.6 & 67.6 & 67.7 & 67.6 & 23.7 \\
\hline 17 & 46.9 & 47.0 & 41.0 & 45.3 & 45.3 & 45.3 & 47.0 \\
\hline 18 & 52.1 & 52.1 & 44.4 & 133.0 & 133.1 & 133.0 & 41.3 \\
\hline 19 & 37.7 & 37.8 & 47.0 & 39.0 & 39.0 & 39.0 & 41.7 \\
\hline 20 & 31.5 & 31.6 & 31.1 & 32.6 & 32.6 & 32.6 & 35.7 \\
\hline 21 & 34.6 & 34.7 & 34.3 & 35.4 & 35.5 & 35.5 & 29.5 \\
\hline 22 & 25.7 & 25.7 & 26.5 & 24.4 & 24.4 & 24.4 & 32.4 \\
\hline 23 & 64.0 & 27.9 & 28.2 & 64.1 & 64.8 & 64.3 & 28.2 \\
\hline 24 & 13.0 & 16.3 & 17.0 & 13.1 & 24.1 & 13.1 & 17.0 \\
\hline 25 & 18.7 & 18.1 & 15.6 & 18.2 & 18.9 & 18.5 & 15.5 \\
\hline 26 & 20.0 & 19.9 & 17.0 & 17.2 & 17.3 & 17.2 & 17.5 \\
\hline 27 & 20.8 & 20.9 & 27.1 & 21.9 & 21.9 & 21.9 & 26.2 \\
\hline 28 & 73.0 & 72.6 & 69.0 & 64.7 & 64.7 & 64.7 & 1765.5 \\
\hline 29 & 33.6 & 33.7 & 32.9 & 25.1 & 25.1 & 25.1 & 28.4 \\
\hline 30 & 23.8 & 23.8 & 24.1 & 32.5 & 32.6 & 64.0 & 65.4 \\
\hline fuc-1 & 105.9 & & & 105.9 & 106.0 & & \\
\hline 2 & 71.5 & & & 71.5 & 71.6 & & \\
\hline 3 & 85.1 & & & 85.2 & 85.4 & & \\
\hline 4 & 71.7 & & & 71.8 & 72.1 & & \\
\hline 5 & 70.9 & & & 71.0 & 71.5 & & \\
\hline 6 & 17.2 & & & 17.2 & 17.4 & & \\
\hline glcI-1 & 106.5 & 106.7 & 106.7 & 106.5 & 106.4 & 106.7 & 95.7 \\
\hline 2 & 75.7 & 75.1 & 75.1 & 75.7 & 75.3 & 75.1 & 74.1 \\
\hline 3 & 78.7 & 76.7 & 76.7 & 78.7 & 78.1 & 76.7 & 79.3 \\
\hline 4 & 72.1 & 79.8 & 79.8 & 72.1 & 75.5 & 79.8 & 71.1 \\
\hline 5 & 78.4 & 75.5 & 75.5 & 78.3 & 78.1 & 75.5 & 78.9 \\
\hline 6 & 62.7 & 69.0 & 68.8 & 62.7 & 64.2 & 69.0 & 62.2 \\
\hline $\mathrm{OCOCH}_{3}$ & & & & 20.1 & & & \\
\hline $\mathrm{OC}^{\mathrm{COCH}}{ }_{3}$ & & & & 170.8 & & & \\
\hline glcII-1 & & 105.0 & 105.0 & & & 105.0 & \\
\hline 2 & & 74.7 & 74.8 & & & 74.7 & \\
\hline 3 & & 78.4 & 78.4 & & & 78.4 & \\
\hline 4 & & 71.4 & 71.4 & & & 71.4 & \\
\hline 5 & & 78.3 & 78.4 & & & 78.3 & \\
\hline 6 & & 62.5 & 62.6 & & & 62.5 & \\
\hline rha-1 & & 102.9 & 102.9 & & & 102.9 & \\
\hline 2 & & 72.5 & 72.6 & & & 72.5 & \\
\hline 3 & & 72.5 & 72.5 & & & 72.5 & \\
\hline 4 & & 73.8 & 73.8 & & & 73.8 & \\
\hline 5 & & 70.5 & 70.4 & & & 70.5 & \\
\hline 6 & & 18.1 & 18.5 & & & 18.1 & \\
\hline
\end{tabular}

NMR spectrum exhibited a total of 36 carbon signals that suggested the presence of one $\beta$-D-glucopyranosyl moiety, the remaining 30 carbon signals were regarded as attributable to a sapogenol moiety. Heteronuclear multiple bond coherence (HMBC) analysis identified six tertiary methyl groups and correlated an oxygen-bearing methylene at $\delta 65.4$ with $\mathrm{H}_{3}-29$; therefore, $\mathrm{CH}_{2} \mathrm{O}$ is present at $\mathrm{C}-30$. This sapogenol is identified as 3 $\beta, 30$-dihydroxyolean-12-ene-28-oic acid, or queretaroic acid. ${ }^{14)} \mathrm{HMBC}$ from the glucosyl anomeric proton was observed at $\mathrm{C}-28$ at $\delta 176.5$. This glycoside was recognized as a new one.

Next, the seeds $(2.6 \mathrm{~kg})$ of $B$. falcatum were crushed with a mixer and extracted by refluxing with $\mathrm{MeOH}$; the resulting extract $(84.7 \mathrm{~g})$ was then separated by using various chromatographies on Diaion HP-20, Sephadex LH-20, silica gel, and preparative thin layer chromatography to provide BFS-1 (30 mg), BFS-8 (8 mg), BFS-9 (13 mg), BFS-10 (31 mg), BFS-11 (34 mg), BFS-13 (28 mg), BFS-14 (15 mg), and BFS-16 (15 mg).

BFS-9 (1), BFS-1 (2), BFS-16 (8), BFS-11 (10), and BFS13 (4) were identified as saikosaponin $\mathrm{a}^{3)}$ saikosaponin $\mathrm{c}^{3)}$ bupleuroside IX, ${ }^{9)}$ saikosaponin $\mathrm{b}_{1}{ }^{2)}$ and saikosaponin $\mathrm{b}_{2}$, respectively, by FAB-MS and ${ }^{1} \mathrm{H}$ - and ${ }^{13} \mathrm{C}-\mathrm{NMR}$ spectra.

BFS-10 (12) was obtained as an amorphous powder having $[\alpha]_{\mathrm{D}}+78.1^{\circ}(\mathrm{MeOH})$. Positive HR-FAB-MS revealed a molecular formula of $\mathrm{C}_{42} \mathrm{H}_{68} \mathrm{O}_{13} \mathrm{Na}$ at $\mathrm{m} / \mathrm{z} 803.4563$ $[\mathrm{M}+\mathrm{Na}]^{+}$. The ${ }^{1} \mathrm{H}-\mathrm{NMR}$ spectrum displayed signals due to six tertiary methyl groups at $\delta 0.89,0.97,1.00,1.28,1.29$, and 1.30 (each $3 \mathrm{H}, \mathrm{s})$; one methyl pentosyl methyl group at $\delta$ $1.44\left(3 \mathrm{H}, \mathrm{d}, J=6.1 \mathrm{~Hz}, \mathrm{H}_{3}-6\right)$; two anomeric protons at $\delta 4.97$ $(1 \mathrm{H}, \mathrm{d}, J=7.9 \mathrm{~Hz})$ and $5.33(1 \mathrm{H}, \mathrm{d}, J=7.9 \mathrm{~Hz})$; and two olefinic protons at $\delta 5.65(1 \mathrm{H}, \mathrm{d}, J=6.1 \mathrm{~Hz})$ and $5.76(1 \mathrm{H}, \mathrm{d}$, $J=6.1 \mathrm{~Hz}$ ). The ${ }^{13} \mathrm{C}-\mathrm{NMR}$ spectrum (Table 3 ) showed the presence of two trisubstituted double bonds at $\delta$ 116.0, 121.1, 145.3, and 154.9; their carbon chemical shifts and proton coupling constants indicated that they were located at the C-ring and homoannular diene, respectively. The respective carbon signals at $\delta 81.5,66.8,64.2$, and 69.3 could be assigned to $\mathrm{C}-3, \mathrm{C}-16, \mathrm{C}-23$, and $\mathrm{C}-28$, respectively, and a $\beta$ configuration was estimated for the hydroxyl group at C-16 by comparing its chemical shift with the reported one. Therefore, sapogenol 27 was determined to be saikogenin $\left.\mathrm{H},{ }^{4}\right)$ which was derived from saikosaponin a by treatment with $1 \mathrm{~N} \mathrm{H}_{2} \mathrm{SO}_{4}$ followed by acid hydrolysis. The sugar moiety was assignable to the glucosyl-( $1 \rightarrow 3)$-fucosyl residue. Consequently, the structure of BFS-10 (12) was determined to be $3-O-\beta$-D-glucopyranosyl- $(1 \rightarrow 3)-\beta$-D-fucopyranosyl $3 \beta, 16 \beta, 23,28$-tetrahydroxyolean-9(11),12(13)-diene; this is identical to that of saikosaponin $\mathrm{g}$, which has been artificially derived from saikosaponin a by treatment with $1 \mathrm{~N} \mathrm{H}_{2} \mathrm{SO}_{4} \cdot{ }^{4}$ )

BFS-8 (9) was obtained as an amorphous powder having $[\alpha]_{\mathrm{D}}-15.0^{\circ}(\mathrm{MeOH})$. Positive HR-FAB-MS revealed a molecular formula of $\mathrm{C}_{30} \mathrm{H}_{48} \mathrm{O}_{4} \mathrm{Na}$ at $\mathrm{m} / z$ 495.3446 [M+Na] ${ }^{+}$. The ${ }^{1} \mathrm{H}-\mathrm{NMR}$ spectrum displayed signals due to six tertiary methyl groups at $\delta 0.93,1.01,1.05,1.07,1.08$, and 1.68 (each $3 \mathrm{H}, \mathrm{s})$, and two olefinic protons at $\delta 5.77(1 \mathrm{H}, \mathrm{d}$, $J=10.7 \mathrm{~Hz})$ and $6.74(1 \mathrm{H}, \mathrm{dd}, J=2.8,10.7 \mathrm{~Hz})$. The ${ }^{13} \mathrm{C}-$ NMR spectrum (Table 3 ) showed a total of 30 carbon signals, including those of a disubstituted double bond and a tetrasubstituted double bond, and four oxygen-bearing carbon signals at $\delta 73.2,67.6,67.7$, and 64.7 , ascribable to C-3, C-16, $\mathrm{C}-23$, and C-28, respectively. The configuration at C-16 in 9 was revealed to be identical with that of saikogenin $\mathrm{D}$, which has been artificially derived from saikosaponin $\mathrm{d}$ by treatment with $1 \mathrm{~N}_{2} \mathrm{SO}_{4}$ followed by acid hydrolysis with $2 \mathrm{~N}$ $\mathrm{H}_{2} \mathrm{SO}_{4} \cdot{ }^{4)}$

BFS-14 (11) was obtained as an amorphous powder having $[\alpha]_{\mathrm{D}}-37.5^{\circ}(\mathrm{MeOH})$. Positive HR-FAB-MS revealed a molecular formula of $\mathrm{C}_{48} \mathrm{H}_{78} \mathrm{O}_{17} \mathrm{Na}$ at $\mathrm{m} / \mathrm{z} 949.5133$ 
Table 2. Oleanene Glycosides Obtained from the Aerial Parts of Bupleurum falcatum

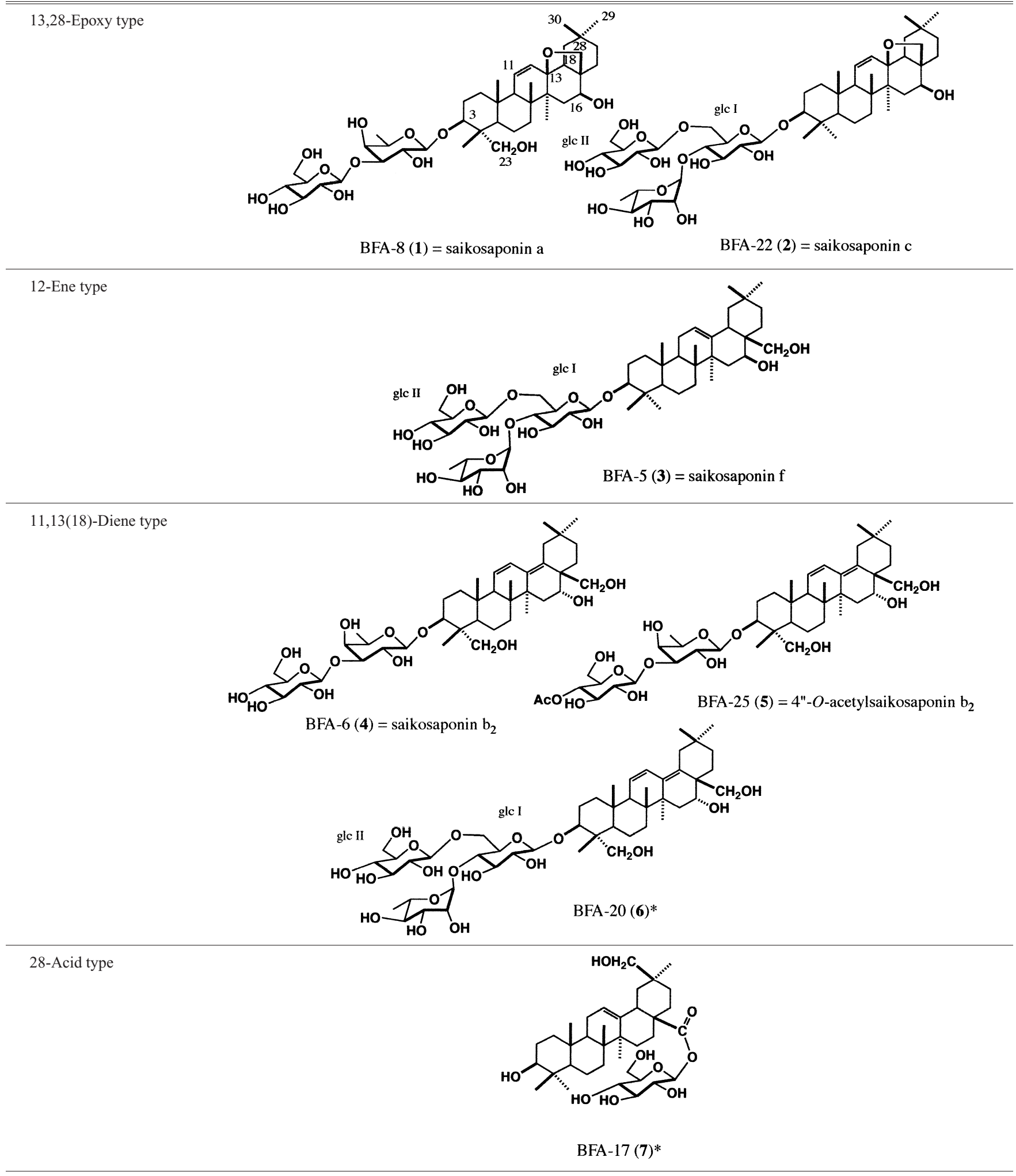

$[\mathrm{M}+\mathrm{Na}]^{+}$. The ${ }^{1} \mathrm{H}-\mathrm{NMR}$ spectrum displayed signals due to seven tertiary methyl groups at $\delta 0.83,0.86,0.90,0.98,0.99$, 1.14 , and 1.32 (each $3 \mathrm{H}, \mathrm{s}$ ); one methyl pentosyl methyl group $\left(3 \mathrm{H}, \mathrm{d}, J=6.1 \mathrm{~Hz}, \mathrm{H}_{3}-6\right)$ at $\delta 1.67$; three anomeric protons at $\delta 4.85(1 \mathrm{H}, \mathrm{d}, J=7.3 \mathrm{~Hz}), 4.98(1 \mathrm{H}, \mathrm{d}, J=7.9 \mathrm{~Hz})$, and $5.87(1 \mathrm{H}$, brs $)$; and two olefinic protons at $\delta 5.64(1 \mathrm{H}, \mathrm{d}$, $J=10.7 \mathrm{~Hz})$ and $6.52(1 \mathrm{H}$, brd, $J=10.7 \mathrm{~Hz})$. The ${ }^{13} \mathrm{C}-\mathrm{NMR}$ spectrum (Table 1) suggested the presence of a heteroannular diene and saikogenin $\mathrm{C}$ as a sapogenol, which is produced from saikosaponin $\mathrm{c}$ by treatment with intestinal bacteria. The sugar moiety was identical with glucosyl- $(1 \rightarrow 6)$-[rhamnosyl- $(1 \rightarrow 4)]$-glucosyl. HMBC analysis indicated that the sugar chain bonding connected with the C-3 of the sapogenol. Therefore, BFS-14 (11) was characterized as 
saikosaponin h, naturally isolated as a genuine saponin for the first time. ${ }^{4}$ )

The aerial parts $(1.4 \mathrm{~kg})$ of $B$. rotundifolium L. were extracted by refluxing with $\mathrm{MeOH}$; the resulting extract $(152 \mathrm{~g})$ was then separated by using the solvent partition method. Various chromatographies on Diaion HP-20, silica gel, and Chromatorex ODS were applied to yield BRA-2 (19 mg), BRA-3 (19 mg), BRA-4 (35 mg), BRA-5 (60 mg), BRA-7 (14 mg), BRA-11 (36 mg), BRA-14 (69 mg), BRA-16

Table $3 .{ }^{13} \mathrm{C}$-Data for Compounds $8-\mathbf{1 2}$

\begin{tabular}{|c|c|c|c|c|c|}
\hline & 8 & 9 & 10 & 11 & 12 \\
\hline C-1 & 40.1 & 38.4 & 38.4 & 38.4 & 37.6 \\
\hline 2 & 27.0 & 27.6 & 26.0 & 26.4 & 26.8 \\
\hline 3 & 88.7 & 73.2 & 81.6 & 89.0 & 81.5 \\
\hline 4 & 43.9 & 43.0 & 43.6 & 39.6 & 43.7 \\
\hline 5 & 55.9 & 48.4 & 47.2 & 55.2 & 43.5 \\
\hline 6 & 18.6 & 18.6 & 18.1 & 18.4 & 17.9 \\
\hline 7 & 33.5 & 31.9 & 32.3 & 32.6 & 32.1 \\
\hline 8 & 41.0 & 41.0 & 40.4 & 40.4 & 43.1 \\
\hline 9 & 52.1 & 53.9 & 54.4 & 54.2 & 154.9 \\
\hline 10 & 38.2 & 36.8 & 36.4 & 36.5 & 38.7 \\
\hline 11 & 76.0 & 126.2 & 127.0 & 126.9 & 116.0 \\
\hline 12 & 122.5 & 126.2 & 125.6 & 125.7 & 121.1 \\
\hline 13 & 148.2 & 136.0 & 136.4 & 136.3 & 145.3 \\
\hline 14 & 43.6 & 41.9 & 44.2 & 44.2 & 43.3 \\
\hline 15 & 36.8 & 32.3 & 34.8 & 34.8 & 36.1 \\
\hline 16 & 66.3 & 67.6 & 76.5 & 76.5 & 66.8 \\
\hline 17 & 39.9 & 45.3 & 44.3 & 44.4 & 40.5 \\
\hline 18 & 44.1 & 133.0 & 133.3 & 133.3 & 133.4 \\
\hline 19 & 47.0 & 39.0 & 38.3 & 38.1 & 46.9 \\
\hline 20 & 31.2 & 32.6 & 32.6 & 32.6 & 31.0 \\
\hline 21 & 34.3 & 35.4 & 35.1 & 35.1 & 34.1 \\
\hline 22 & 26.0 & 24.4 & 29.9 & 29.9 & 26.1 \\
\hline 23 & 28.2 & 67.7 & 63.9 & 27.9 & 64.2 \\
\hline 24 & 17.4 & 12.6 & 13.0 & 16.4 & 13.7 \\
\hline 25 & 18.3 & 18.7 & 18.7 & 18.4 & 21.0 \\
\hline 26 & 17.3 & 17.2 & 17.2 & 16.9 & 21.2 \\
\hline 27 & 26.3 & 21.9 & 21.9 & 21.9 & 26.1 \\
\hline 28 & 68.6 & 64.7 & 63.9 & 63.9 & 69.3 \\
\hline 29 & 33.1 & 25.1 & 24.7 & 24.8 & 33.1 \\
\hline 30 & 24.0 & 32.5 & 32.2 & 32.2 & 24.0 \\
\hline $\mathrm{OC}_{3}$ & 54.1 & & & & \\
\hline fuc-1 & 106.9 & & 105.9 & & 105.9 \\
\hline 2 & 71.6 & & 71.5 & & 71.5 \\
\hline 3 & 85.3 & & 85.1 & & 85.2 \\
\hline 4 & 72.2 & & 72.1 & & 72.0 \\
\hline 5 & 71.0 & & 70.1 & & 70.9 \\
\hline 6 & 17.0 & & 17.0 & & 17.2 \\
\hline glcI-1 & 106.7 & & 106.4 & 106.7 & 106.5 \\
\hline 2 & 75.9 & & 75.7 & 75.2 & 75.7 \\
\hline 3 & 78.7 & & 78.6 & 76.7 & 78.7 \\
\hline 4 & 71.4 & & 71.7 & 79.8 & 71.7 \\
\hline 5 & 78.1 & & 78.3 & 75.5 & 78.3 \\
\hline 6 & 62.7 & & 62.6 & 69.0 & 62.6 \\
\hline glcII-1 & & & & 105.0 & \\
\hline 2 & & & & 74.8 & \\
\hline 3 & & & & 78.4 & \\
\hline 4 & & & & 71.5 & \\
\hline 5 & & & & 78.3 & \\
\hline 6 & & & & 62.6 & \\
\hline rha-1 & & & & 102.9 & \\
\hline 2 & & & & 72.5 & \\
\hline 3 & & & & 72.6 & \\
\hline 4 & & & & 73.8 & \\
\hline 5 & & & & 70.5 & \\
\hline 6 & & & & 18.2 & \\
\hline
\end{tabular}

(12 mg), BRA-17 (14 mg), BRA-22 (22 mg), BRA-18 (38 mg), BRA-19 (6 mg), and BRA-20 (5 mg).

BRA-3 (13), BRA-5 (14), BRA-2 (15), BRA-4 (16), and BRA-7 (17) were identified as rotundifolioside $\mathrm{J},{ }^{11,12}$ ) rotundifolioside $\mathrm{I}^{11,12)}$ rotundioside $\mathrm{E},{ }^{15,16)}$ and rotundioside $\mathrm{W},{ }^{11,12)}$ and rotundioside $\mathrm{V}^{11,12)}$ respectively.

BRA-22 (18) was obtained as an amorphous powder having $[\alpha]_{\mathrm{D}}-22.5^{\circ}(\mathrm{MeOH})$. Positive HR-FAB-MS revealed a molecular formula of $\mathrm{C}_{48} \mathrm{H}_{78} \mathrm{O}_{17} \mathrm{Na}$ at $\mathrm{m} / \mathrm{z} 949.5143$ $[\mathrm{M}+\mathrm{Na}]^{+}$. The ${ }^{1} \mathrm{H}-\mathrm{NMR}$ spectrum displayed signals due to six tertiary methyl groups at $\delta 0.87,0.91,1.09,1.25,1.37$, and 1.77 (each $3 \mathrm{H}, \mathrm{s})$; two methyl pentosyl methyl groups at $\delta 1.52\left(3 \mathrm{H}, \mathrm{d}, J=6.7 \mathrm{~Hz}, \mathrm{H}_{3}-6\right)$ and $1.86(3 \mathrm{H}, \mathrm{d}, J=6.1 \mathrm{~Hz}$, $\left.\mathrm{H}_{3}-6\right)$; three anomeric protons at $\delta 4.79(1 \mathrm{H}, \mathrm{d}, J=7.3 \mathrm{~Hz})$, $5.69(1 \mathrm{H}, \mathrm{d}, J=7.3 \mathrm{~Hz})$, and $6.44(1 \mathrm{H}, \mathrm{br} \mathrm{s})$; and two olefinic protons at $\delta 5.70(1 \mathrm{H}, \mathrm{d}, J=7.9 \mathrm{~Hz})$ and $6.77(1 \mathrm{H}, \mathrm{brd}$, $J=7.9 \mathrm{~Hz}$ ). The ${ }^{13} \mathrm{C}-\mathrm{NMR}$ spectrum (Table 5) showed a total of 48 carbon signals, of which 18 indicated the presence of a rhamnosyl-( $1 \rightarrow 2)$-glucosyl-( $1 \rightarrow 2)$-fucosyl moiety identical to that of BRA-3 (13). The remaining signals showed good agreement with those of rotundiogenin $\mathrm{B}$ in addition to those of C-20, C-29, and C-30. This means that in 18, a hydroxymethyl is present at $\delta 73.6$ at $\mathrm{C}-29$ or $\mathrm{C}-30$. HMBC analysis also supported this conclusion. By comparison with the $3 \beta, 16 \alpha, 28,30$-tetrahydroxyolean-11,13(18)-dine derivative ${ }^{17)}$ isolated from $B$. spinosum and the $30-\mathrm{CH}_{2} \mathrm{OH}-$ isomer $^{18)}$ isolated from $B$. rigidum, 18 was determined to be a C-30-hydroxymethyl derivative. Therefore, its structure was characterized as 3-O- $\alpha$-L-rhamnopyranosyl- $(1 \rightarrow 2)-\beta$-D-glucopyranosyl-( $1 \rightarrow 2)$ - $\beta$-D-fucopyranosyl $3 \beta, 16,28,30$-tetrahydroxyolean-11,13(18)-diene.

BRA-11 (19) was obtained as an amorphous powder having $[\alpha]_{\mathrm{D}}-41.8^{\circ}(\mathrm{MeOH})$. Positive HR-FAB-MS revealed a molecular formula of $\mathrm{C}_{48} \mathrm{H}_{78} \mathrm{O}_{17} \mathrm{Na}$ at $\mathrm{m} / \mathrm{z} \quad 949.5139$ $[\mathrm{M}+\mathrm{Na}]^{+}$. The ${ }^{1} \mathrm{H}-\mathrm{NMR}$ spectrum displayed signals due to seven tertiary methyl groups at $\delta 0.87,0.92,1.09,1.30,1.34$, 1.38 , and 1.77 (each $3 \mathrm{H}, \mathrm{s}$ ); two methyl pentosyl methyl groups at $\delta 1.53\left(3 \mathrm{H}, \mathrm{d}, J=6.1 \mathrm{~Hz}, \mathrm{H}_{3}-6\right)$ and $1.87(3 \mathrm{H}, \mathrm{d}$, $\left.J=6.1 \mathrm{~Hz}, \mathrm{H}_{3}-6\right)$; one oxygen-bearing methine proton at $\delta$ $3.33(1 \mathrm{H}$, brd, $J=8.0 \mathrm{~Hz})$; one oxygen-bearing methylene proton at $\delta 3.85(1 \mathrm{H}, \mathrm{d}, J=10.9 \mathrm{~Hz})$ and 4.33 (overlapped); three anomeric protons at $\delta 4.80(1 \mathrm{H}, \mathrm{d}, J=6.7 \mathrm{~Hz}), 5.70$ $(1 \mathrm{H}, \mathrm{d}, J=7.3 \mathrm{~Hz})$, and $6.44(1 \mathrm{H}$, br s); and two olefinic protons at $\delta 5.73(1 \mathrm{H}, \mathrm{d}, J=10.4 \mathrm{~Hz})$ and $6.77(1 \mathrm{H}$, brd, $J=10.4 \mathrm{~Hz}$ ). The ${ }^{13} \mathrm{C}-\mathrm{NMR}$ spectrum (Table 5) showed a total of 48 carbon signals, of which 18 revealed the presence of a rhamnosyl- $(1 \rightarrow 2)$-glucosyl- $(1 \rightarrow 2)$-fucosyl moiety, as in BRA-22 (18). In the remaining signals due to the sapogenol

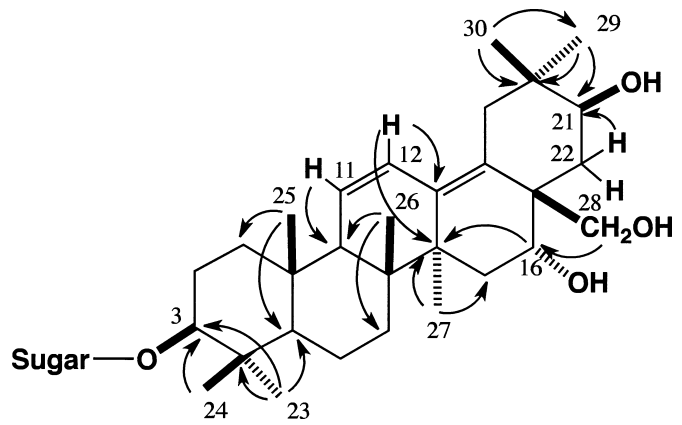

Fig. 1. HMBC of BRA-11 (19) 
Table 4. Oleanene Glycosides Obtained from the Seeds of Bupleurum falcatum

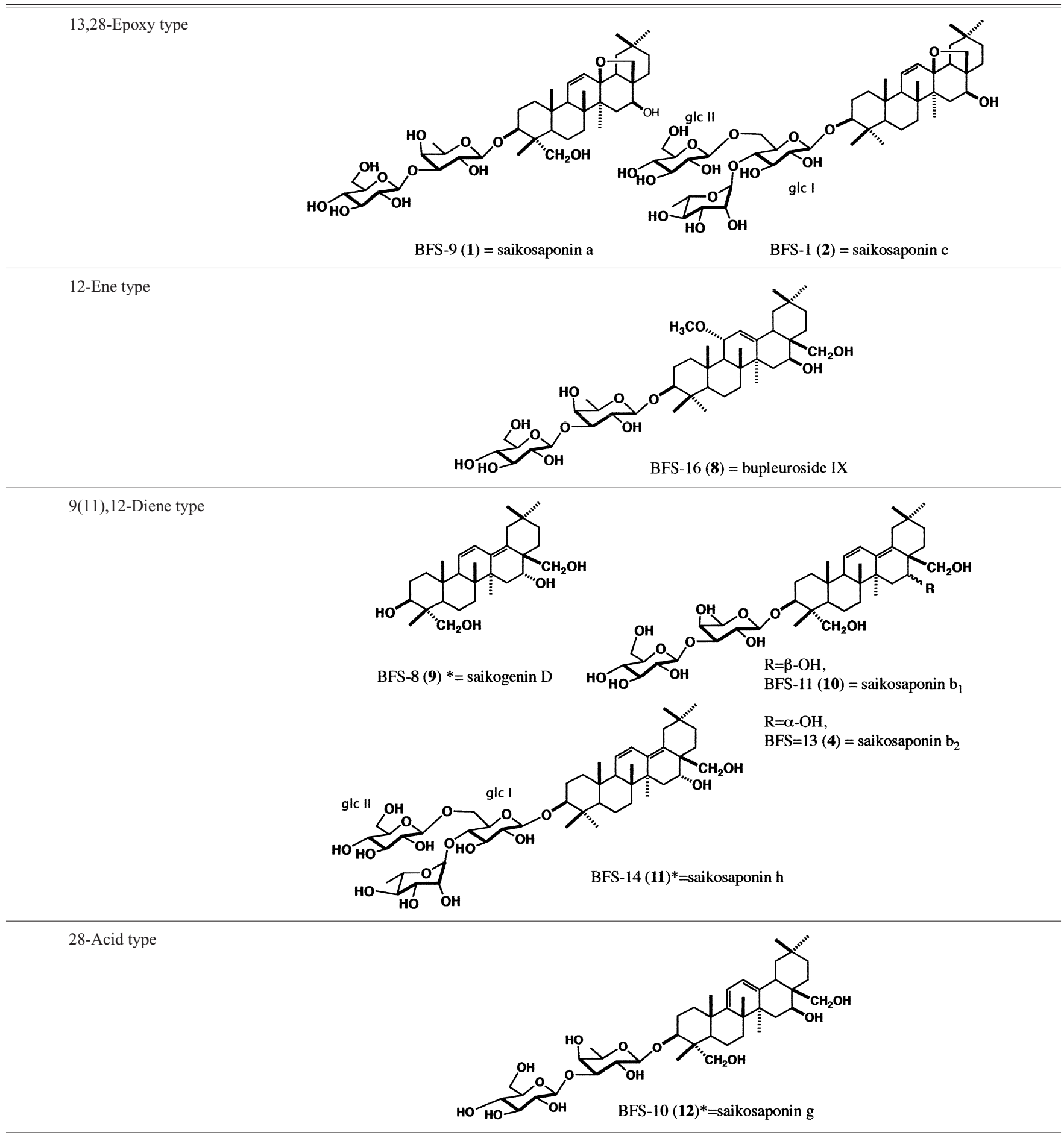

moiety, three oxygen-bearing carbon signals were observed; the signals at $\delta 67.3$ and 89.5 were assigned to carbons having an $\alpha$-hydroxyl group at C-16 and a $\beta$-hydroxyl group at $\mathrm{C}-3$, respectively. The remaining signal, at $\delta 73.7$, was assigned to $\mathrm{C}-21$ because HMBC analysis exhibited correlations from $\mathrm{H}_{3}-29$ and $\mathrm{H}_{3}-30$ to $\mathrm{C}-22$, and from $\mathrm{H}_{2}-22$ to $\mathrm{C}-22$ (Fig. 1). Here, the configuration at the $\mathrm{C}-21$ hydroxyl group was deduced to be $\beta$-equatorial because the signals due to $\mathrm{H}_{3}-29$ and $\mathrm{H}_{3}-30$ in BRA-11 (19) were shifted upward relative to those of rotundiogenin $\mathrm{C}$, which has a C-21 hydroxyl group,${ }^{19)}$ by the influence of the hydroxyl group. Therefore, the structure of BRA-11 (19) was characterized as 3-O- $\alpha$-Lrhamnopyranosyl-( $1 \rightarrow 2)$ - $\beta$-D-glucopyranosyl-( $1 \rightarrow 2)$ - $\beta$-Dfucopyranosyl $3 \beta, 16 \alpha, 21 \beta, 28$-tetrahydroxyolean-11,13(18)diene, which indicates a new sapogenol.

BRA-16 (20) was obtained as an amorphous powder having $[\alpha]_{\mathrm{D}}-1.5^{\circ}(\mathrm{MeOH})$. Positive HR-FAB-MS revealed a molecular formula of $\mathrm{C}_{47} \mathrm{H}_{76} \mathrm{O}_{17} \mathrm{Na}$ at $\mathrm{m} / \mathrm{z} 935.4973$ $[\mathrm{M}+\mathrm{Na}]^{+}$. The ${ }^{1} \mathrm{H}-\mathrm{NMR}$ spectrum showed signals due to seven tertiary methyl groups at $\delta 0.88,0.94,1.11,1.30,1.31$, 1.34 , and 1.76 (each $3 \mathrm{H}, \mathrm{s}$ ); one methyl pentosyl methyl groups at $\delta 1.59\left(3 \mathrm{H}, \mathrm{d}, J=6.1 \mathrm{~Hz}, \mathrm{H}_{3}-6\right)$ and 1.87 ; one oxy- 
Table $5 .{ }^{13} \mathrm{C}$-Data for Compounds $\mathbf{1 3}-\mathbf{1 9}$

\begin{tabular}{|c|c|c|c|c|c|c|c|}
\hline & 13 & 14 & 15 & 16 & 17 & 18 & 19 \\
\hline C-1 & 38.7 & 38.7 & 38.4 & 38.5 & 38.4 & 38.4 & 38.4 \\
\hline 2 & 26.6 & 26.6 & 26.6 & 26.6 & 26.0 & 26.6 & 26.6 \\
\hline 3 & 89.5 & 88.8 & 89.5 & 88.8 & 84.6 & 89.6 & 89.5 \\
\hline 4 & 39.9 & 39.8 & 40.0 & 39.8 & 43.7 & 39.9 & 39.9 \\
\hline 5 & 55.6 & 55.5 & 55.6 & 55.5 & 48.4 & 55.6 & 55.5 \\
\hline 6 & 18.0 & 18.0 & 18.6 & 18.6 & 18.5 & 18.7 & 18.6 \\
\hline 7 & 32.0 & 32.0 & 32.6 & 32.6 & 32.4 & 32.6 & 32.5 \\
\hline 8 & 42.0 & 42.0 & 42.0 & 41.1 & 41.9 & 42.0 & 41.1 \\
\hline 9 & 52.7 & 52.6 & 53.8 & 53.8 & 54.0 & 53.9 & 53.8 \\
\hline 10 & 36.4 & 36.3 & 36.6 & 36.6 & 36.6 & 36.6 & 36.6 \\
\hline 11 & 134.2 & 134.1 & 126.3 & 126.2 & 126.3 & 126.4 & 126.5 \\
\hline 12 & 129.1 & 129.1 & 126.2 & 126.3 & 126.2 & 126.1 & 126.4 \\
\hline 13 & 85.1 & 85.1 & 136.0 & 135.1 & 136.1 & 136.4 & 136.3 \\
\hline 14 & 44.1 & 44.1 & 41.1 & 41.9 & 41.1 & 41.1 & 31.7 \\
\hline 15 & 35.6 & 35.5 & 31.9 & 31.9 & 31.9 & 32.1 & 67.3 \\
\hline 16 & 77.1 & 76.9 & 67.7 & 67.7 & 67.7 & 68.0 & 47.7 \\
\hline 17 & 46.4 & 46.4 & 45.3 & 45.3 & 45.3 & 45.9 & 47.8 \\
\hline 18 & 61.5 & 61.5 & 133.1 & 133.1 & 133.1 & 133.0 & 132.2 \\
\hline 19 & 38.5 & 38.3 & 39.0 & 39.1 & 39.1 & 34.0 & 39.3 \\
\hline 20 & 41.7 & 41.7 & 32.6 & 32.6 & 32.6 & 38.3 & 38.0 \\
\hline 21 & 33.7 & 33.7 & 35.5 & 35.5 & 35.5 & 30.3 & 73.7 \\
\hline 22 & 35.4 & 35.4 & 24.5 & 24.5 & 24.5 & 24.3 & 33.1 \\
\hline 23 & 28.1 & 17.9 & 28.1 & 28.0 & 65.6 & 28.2 & 28.1 \\
\hline 24 & 16.2 & 16.3 & 16.3 & 16.4 & 12.9 & 16.3 & 16.3 \\
\hline 25 & 17.4 & 18.3 & 18.3 & 18.4 & 18.9 & 18.3 & 18.3 \\
\hline 26 & 19.0 & 19.4 & 17.4 & 17.3 & 17.3 & 17.4 & 17.2 \\
\hline 27 & 16.4 & 16.4 & 21.9 & 21.9 & 21.9 & 22.0 & 21.9 \\
\hline 28 & 77.7 & 77.7 & 64.8 & 64.8 & 64.8 & 65.1 & 65.7 \\
\hline 29 & 18.9 & 18.9 & 25.1 & 25.1 & 25.1 & 21.1 & 29.2 \\
\hline 30 & 19.8 & 19.8 & 32.6 & 32.6 & 32.6 & 73.6 & 18.4 \\
\hline fuc-1 & 105.3 & 105.3 & 105.3 & 105.2 & 103.7 & 105.3 & 105.3 \\
\hline 2 & 77.2 & 80.6 & 77.4 & 80.6 & 81.2 & 77.6 & 77.3 \\
\hline 3 & 76.3 & 75.5 & 76.2 & 75.5 & 75.6 & 76.3 & 76.2 \\
\hline 4 & 72.8 & 72.6 & 72.9 & 72.6 & 72.6 & 72.9 & 72.9 \\
\hline 5 & 70.9 & 71.2 & 70.8 & 71.2 & 71.2 & 70.9 & 70.8 \\
\hline 6 & 19.4 & 17.3 & 17.2 & 17.2 & 17.3 & 17.2 & 17.4 \\
\hline glc-1 & 102.2 & 103.1 & 102.2 & 103.2 & 103.4 & 102.2 & 102.2 \\
\hline 2 & 78.1 & 84.7 & 78.1 & 84.8 & 84.6 & 78.1 & 78.1 \\
\hline 3 & 79.5 & 77.7 & 79.5 & 78.0 & 77.9 & 79.5 & 79.5 \\
\hline 4 & 73.0 & 71.8 & 72.8 & 71.8 & 71.1 & 73.0 & 72.9 \\
\hline 5 & 76.9 & 77.5 & 77.0 & 77.7 & 77.7 & 77.3 & 77.1 \\
\hline 6 & 63.3 & 62.8 & 63.3 & 62.8 & 62.4 & 63.3 & 63.3 \\
\hline rha-1 & 101.9 & & 101.9 & & & 101.9 & 101.9 \\
\hline 2 & 72.5 & & 72.5 & & & 72.5 & 72.8 \\
\hline 3 & 72.8 & & 72.8 & & & 72.8 & 72.5 \\
\hline 4 & 74.4 & & 74.3 & & & 74.4 & 74.3 \\
\hline 5 & 69.5 & & 69.5 & & & 69.5 & 69.5 \\
\hline 6 & 18.2 & & 19.0 & & & 19.0 & 19.0 \\
\hline xyl-1 & & 106.5 & & 106.6 & 106.5 & & \\
\hline 2 & & 75.8 & & 75.9 & 75.9 & & \\
\hline 3 & & 78.0 & & 77.5 & 77.6 & & \\
\hline 4 & & 70.8 & & 70.8 & 70.8 & & \\
\hline 5 & & 67.4 & & 67.4 & 67.5 & & \\
\hline
\end{tabular}

gen-bearing methine proton at $\delta 3.33(1 \mathrm{H}$, br d, $J=8.0 \mathrm{~Hz})$; one oxygenated methylene proton at $\delta 3.85(1 \mathrm{H}, \mathrm{d}$, $J=10.9 \mathrm{~Hz})$, three anomeric protons at $\delta 4.83(1 \mathrm{H}, \mathrm{d}$, $J=7.9 \mathrm{~Hz}), 5.37(1 \mathrm{H}, \mathrm{d}, J=7.0 \mathrm{~Hz})$, and $5.42(1 \mathrm{H}, \mathrm{d}$, $J=7.3 \mathrm{~Hz})$; and two olefinic protons at $\delta 5.73(1 \mathrm{H}, \mathrm{d}$, $J=10.4 \mathrm{~Hz})$ and $6.78(1 \mathrm{H}$, brd, $J=10.4 \mathrm{~Hz})$. The ${ }^{13} \mathrm{C}-\mathrm{NMR}$ spectrum (Table 5) showed a total of 47 carbon signals, which indicated the presence of $3 \beta, 16 \alpha, 21 \beta, 28$-tetrahydroxyolean-11,13(18)-diene, as in BRA-11 (19), and a xylosyl$(1 \rightarrow 2)$-glucosyl-( $1 \rightarrow 2)$-fucosyl moiety, as in BRA-5 (14),
Table $6 .{ }^{13} \mathrm{C}$-Data for Compounds $\mathbf{2 0}-\mathbf{2 5}$

\begin{tabular}{|c|c|c|c|c|c|c|}
\hline & 20 & 21 & 22 & 23 & 24 & 25 \\
\hline C-1 & 38.4 & 38.5 & 38.7 & 38.9 & 38.8 & 38.8 \\
\hline 2 & 26.6 & 26.6 & 26.4 & 26.4 & 26.4 & 26.4 \\
\hline 3 & 88.8 & 89.5 & 89.8 & 89.9 & 89.7 & 89.7 \\
\hline 4 & 39.8 & 39.9 & 39.6 & 40.0 & 39.6 & 39.6 \\
\hline 5 & 55.5 & 55.6 & 56.0 & 56.2 & 56.0 & 56.0 \\
\hline 6 & 18.6 & 18.6 & 18.5 & 18.4 & 18.5 & 18.5 \\
\hline 7 & 32.6 & 32.4 & 32.3 & 32.3 & 32.1 & 31.6 \\
\hline 8 & 41.1 & 42.3 & 40.0 & 40.1 & 40.0 & 40.1 \\
\hline 9 & 53.8 & 54.0 & 48.0 & 48.2 & 47.1 & 47.1 \\
\hline 10 & 36.6 & 36.7 & 36.9 & 37.0 & 37.0 & 36.9 \\
\hline 11 & 126.4 & 126.0 & 23.8 & 23.8 & 23.8 & 23.8 \\
\hline 12 & 126.4 & 128.0 & 122.5 & 122.6 & 122.6 & 122.1 \\
\hline 13 & 136.3 & 137.0 & 144.6 & 144.5 & 144.4 & 145.0 \\
\hline 14 & 41.9 & 40.9 & 42.1 & 42.1 & 42.0 & 41.9 \\
\hline 15 & 31.7 & 32.3 & 29.3 & 29.4 & 35.9 & 36.0 \\
\hline 16 & 67.3 & 68.4 & 23.0 & 22.9 & 74.1 & 73.9 \\
\hline 17 & 47.8 & 46.0 & 47.0 & 47.0 & 49.1 & 49.1 \\
\hline 18 & 132.2 & 131.0 & 41.7 & 41.9 & 41.3 & 41.3 \\
\hline 19 & 39.4 & 39.3 & 40.2 & 46.2 & 47.2 & 47.3 \\
\hline 20 & 38.0 & 32.6 & 30.7 & 30.7 & 30.8 & 30.8 \\
\hline 21 & 73.7 & 217.1 & 34.0 & 34.0 & 36.1 & 36.6 \\
\hline 22 & 33.2 & 44.3 & 33.2 & 33.5 & 33.4 & 33.5 \\
\hline 23 & 28.0 & 28.2 & 28.6 & 28.6 & 28.3 & 28.5 \\
\hline 24 & 16.4 & 16.3 & 16.8 & 16.7 & 16.8 & 16.8 \\
\hline 25 & 18.4 & 18.4 & 15.4 & 15.5 & 15.6 & 15.6 \\
\hline 26 & 17.2 & 17.4 & 17.4 & 17.5 & 17.5 & 17.5 \\
\hline 27 & 21.9 & 22.2 & 26.2 & 26.2 & 27.2 & 27.2 \\
\hline 28 & 65.8 & 69.5 & 176.5 & 176.5 & 175.5 & 175.9 \\
\hline 29 & 29.3 & 26.1 & 23.7 & 23.7 & 24.6 & 24.6 \\
\hline 30 & 18.4 & 24.6 & 33.1 & 33.2 & 33.2 & 33.2 \\
\hline fuc-1 & 105.0 & 105.3 & & & & \\
\hline 2 & 80.7 & 77.4 & & & & \\
\hline 3 & $\begin{array}{l}00.1 \\
75.5\end{array}$ & 76.3 & & & & \\
\hline 4 & 72.6 & 73.0 & & & & \\
\hline 5 & 71.2 & 70.9 & & & & \\
\hline 6 & 17.3 & 17.3 & & & & \\
\hline glcI-1 & 103.2 & 102.2 & 105.2 & 105.2 & 105.0 & 105.2 \\
\hline 2 & 84.8 & 78.1 & 78.5 & 78.5 & 78.3 & 78.4 \\
\hline 3 & 78.0 & 79.5 & 79.5 & 78.8 & 78.9 & 78.8 \\
\hline 4 & 71.8 & 72.8 & 70.1 & 70.1 & 72.5 & 72.6 \\
\hline 5 & 77.7 & 77.0 & 77.9 & 77.9 & 77.8 & 77.8 \\
\hline 6 & 62.8 & 63.3 & 62.8 & 62.8 & 62.6 & 62.8 \\
\hline glcII-1 & & & 93.4 & 93.5 & 95.8 & 93.8 \\
\hline 2 & & & 78.4 & 78.4 & 74.2 & 78.2 \\
\hline 3 & & & 79.4 & 79.2 & 79.4 & 79.3 \\
\hline 4 & & & 78.8 & 79.5 & 78.8 & 79.9 \\
\hline 5 & & & 79.2 & 79.4 & 79.3 & 79.3 \\
\hline 6 & & & 61.9 & 61.9 & 62.1 & 61.8 \\
\hline glcIII-1 & & & 101.9 & 102.0 & 101.9 & 101.9 \\
\hline 2 & & & 75.9 & 75.9 & 76.0 & 75.9 \\
\hline 3 & & & 77.9 & 77.9 & 78.3 & 77.9 \\
\hline 4 & & & 72.8 & 72.8 & 72.8 & 72.8 \\
\hline 5 & & & 77.5 & 77.5 & 77.4 & 77.5 \\
\hline 6 & & & 63.9 & 63.4 & 63.4 & 63.3 \\
\hline glcIV-1 & & & 104.6 & 105.2 & & 105.1 \\
\hline 2 & & & 75.9 & 76.7 & & 75.8 \\
\hline 3 & & & 79.5 & 79.6 & & 79.4 \\
\hline 4 & & & 72.0 & 72.1 & & 71.5 \\
\hline 5 & & & 78.2 & 78.6 & & 78.2 \\
\hline 6 & & & 62.8 & 62.8 & & 62.7 \\
\hline glcV-1 & & & & 102.4 & & \\
\hline 2 & & & & 78.6 & & \\
\hline 3 & & & & 79.2 & & \\
\hline 4 & & & & 72.4 & & \\
\hline 5 & & & & 77.1 & & \\
\hline 6 & & & & 63.7 & & \\
\hline xyl-1 & 106.6 & & & & & \\
\hline 2 & 75.9 & & & & & \\
\hline 3 & 77.4 & & & & & \\
\hline 4 & 70.8 & & & & & \\
\hline 5 & 67.4 & & & & & \\
\hline rha-1 & & 101.9 & 101.8 & 101.8 & 101.9 & 101.8 \\
\hline 2 & & 72.6 & 72.4 & 71.7 & 71.8 & 71.9 \\
\hline 3 & & 72.8 & 72.6 & 72.7 & 72.3 & 72.8 \\
\hline 4 & & 74.3 & 74.4 & 74.4 & 74.4 & 74.3 \\
\hline 5 & & 69.6 & 69.5 & 69.5 & 69.5 & 69.5 \\
\hline 6 & & 19.1 & 19.1 & 19.1 & 18.9 & 19.0 \\
\hline
\end{tabular}


Table 7. Oleanene Glycosides Obtained from the Aerial Parts of Bupleurum rotundifolium

13,28-Epoxy type

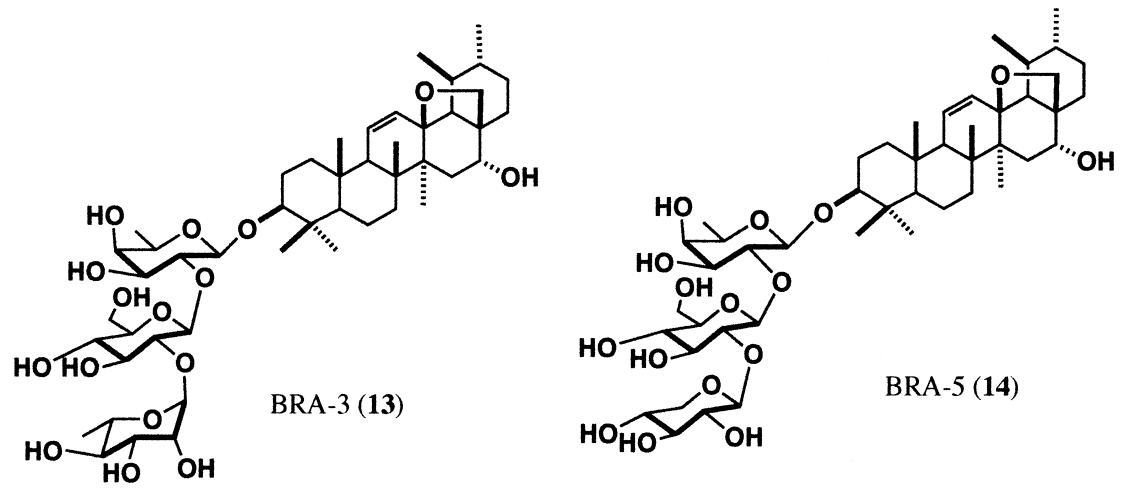

11,13(18)-Diene type

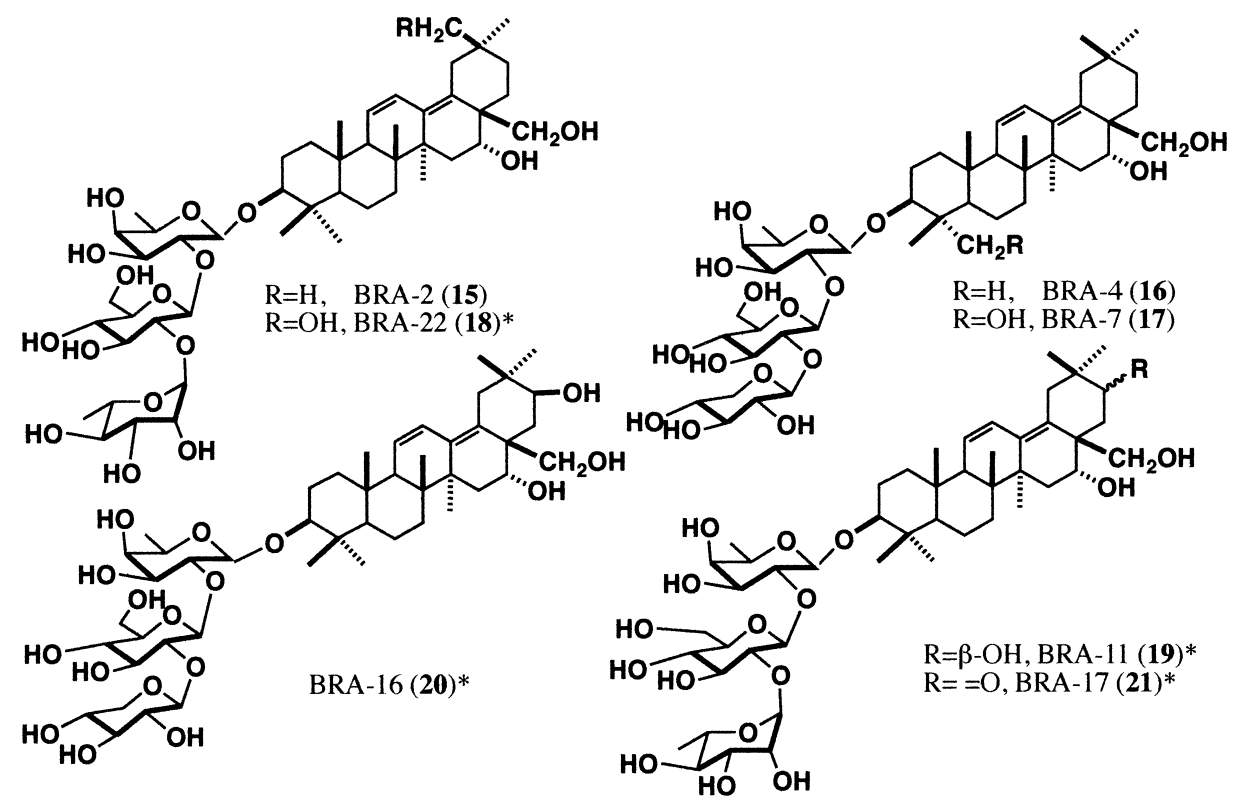

28-Acid type

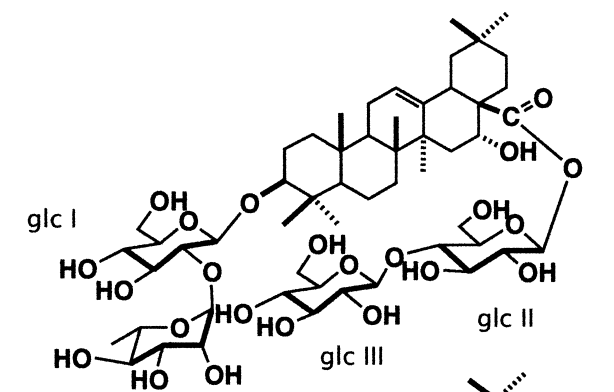

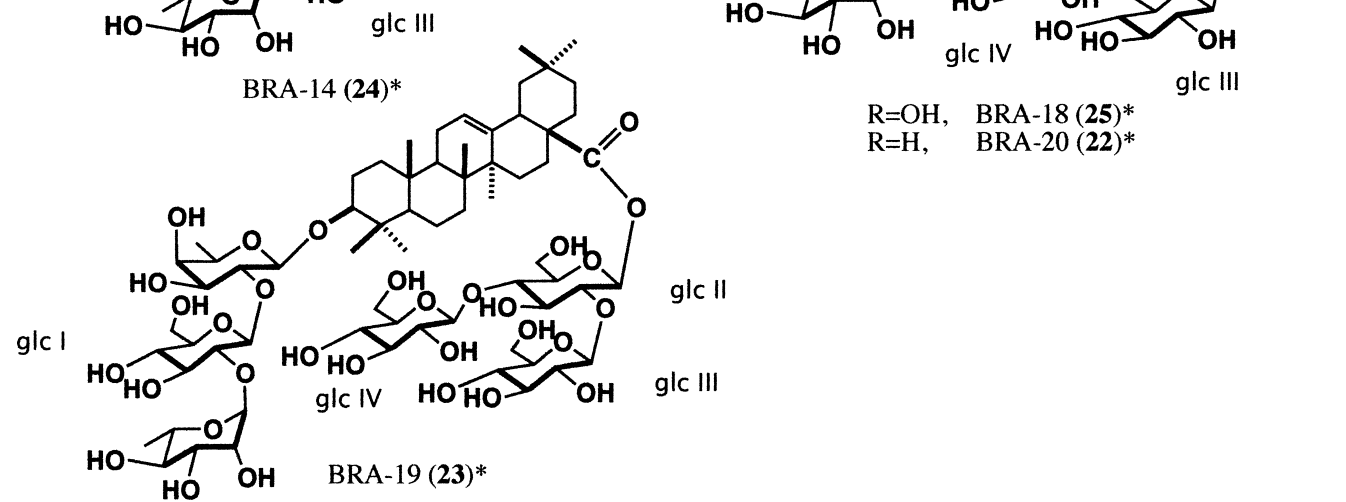


BRA-4 (16), and BRA-7 (17). Therefore, the structure of BRA-16 (20) was characterized as 3- $O$ - $\beta$-D-xylopyranosyl$(1 \rightarrow 2)$ - $\beta$-D-glucopyranosyl- $(1 \rightarrow 2)-\beta$-D-fucopyranosyl $3 \beta, 16 \alpha, 21 \beta, 28$-tetrahydroxyolean-11,13(18)-diene, which indicates a new sapogenol.

BRA-17 (21) was obtained as an amorphous powder having $[\alpha]_{\mathrm{D}}+11.6^{\circ}(\mathrm{MeOH})$. Positive HR-FAB-MS revealed a molecular formula of $\mathrm{C}_{48} \mathrm{H}_{76} \mathrm{O}_{17} \mathrm{Na}$ at $\mathrm{m} / \mathrm{z} \quad 947.4987$ $[\mathrm{M}+\mathrm{Na}]^{+}$. The ${ }^{1} \mathrm{H}-\mathrm{NMR}$ spectrum showed signals due to seven tertiary methyl groups at $\delta 0.83,0.90,1.10,1.25,1.32$, 1.39 , and 1.72 (each $3 \mathrm{H}, \mathrm{s}$ ); two methyl pentosyl methyl groups at $\delta 1.52\left(3 \mathrm{H}, \mathrm{d}, J=6.1 \mathrm{~Hz}, \mathrm{H}_{3}-6\right)$ and $1.88(3 \mathrm{H}, \mathrm{d}$, $\left.J=6.1 \mathrm{~Hz}, \mathrm{H}_{3}-6\right)$; two methylene protons at $\delta 2.67$ and 2.96 $(2 \mathrm{H}, \mathrm{ABq}, J=14.0 \mathrm{~Hz})$ and 3.24 and $3.49(2 \mathrm{H}, \mathrm{ABq}$, $J=5.3 \mathrm{~Hz}$ ); one oxygen-bearing methine proton at $\delta 3.34$ $(1 \mathrm{H}, \mathrm{m})$; one oxygen-bearing methylene proton at $\delta 3.88$, $4.04(2 \mathrm{H}, \mathrm{ABq}, J=11.0 \mathrm{~Hz})$; two olefinic protons at $\delta 5.77$ $(1 \mathrm{H}, \mathrm{d}, J=11.0 \mathrm{~Hz})$ and $6.71(1 \mathrm{H}, \mathrm{brd}, J=11.0 \mathrm{~Hz})$; and three anomeric protons at $\delta 4.81(1 \mathrm{H}, \mathrm{d}, J=7.3 \mathrm{~Hz}), 5.72(1 \mathrm{H}, \mathrm{d}$, $J=7.9 \mathrm{~Hz})$, and $6.44(1 \mathrm{H}, \mathrm{brs})$.

The ${ }^{13} \mathrm{C}$-NMR spectrum (Table 5) showed the presence of the same sugar moiety as in BRA-11 (19) and the occurrence of a carbonyl group at $\delta 217.1$ in the sapogenol moiety. HMBC appeared from $\mathrm{H}_{3}-29, \mathrm{H}_{3}-30$, and $\mathrm{H}_{2}-22$ to the carbon at $\delta$ 217.1; therefore, its carbonyl carbon should be located at C-21. Consequently, the structure of BRA-17 (21) was characterized as 3-O- $\alpha$-L-rhamnopyranosyl- $(1 \rightarrow 2)-\beta$-D-glucopyranosyl- $(1 \rightarrow 2)$ - $\beta$-D-fucopyranosyl $3 \beta, 16 \alpha, 28$-trihydroxyolean-11,13(18)-diene-21-one.

BRA-20 (22) was obtained as an amorphous powder having $[\alpha]_{\mathrm{D}}+34.0^{\circ}(\mathrm{MeOH})$. Positive HR-FAB-MS revealed a molecular formula of $\mathrm{C}_{60} \mathrm{H}_{98} \mathrm{O}_{27} \mathrm{Na}$ at $\mathrm{m} / \mathrm{z} 1273.6198$ $[\mathrm{M}+\mathrm{Na}]^{+}$. The ${ }^{1} \mathrm{H}-\mathrm{NMR}$ spectrum showed signals due to seven tertiary methyl groups at $\delta 0.83,0.87,0.89,1.00,1.24$, 1.28 , and 1.31 (each $3 \mathrm{H}, \mathrm{s}$ ); one methyl pentosyl methyl

Table 8. Hepatoprotective and Hepatotoxic Activity of 13,28-Epoxy Type Saponins

\begin{tabular}{cccc}
\hline \hline Substances & Dose $(\mu \mathrm{M})$ & Protection $(\%)$ & Toxicity $(\%)$ \\
\hline 1 (Saikosaponin a) & 10 & 13 & 96 \\
& 30 & -8 & $317^{\dagger}$ \\
2 (Saikosaponin c) & 200 & $-33^{\dagger}$ & $492^{\dagger}$ \\
& 500 & $-20^{\dagger}$ & $455^{\dagger}$ \\
& 10 & $13^{*}$ & $390^{\dagger}$ \\
& 30 & 2 & $\mathrm{ND}$ \\
& 90 & $13^{*}$ & $\mathrm{ND}$ \\
& 200 & $20^{* * *}$ & $\mathrm{ND}$ \\
& 500 & $42^{* * *}$ & $\mathrm{ND}$ \\
& 10 & 0 & 110 \\
& 30 & $-50^{\dagger}$ & $605^{\dagger}$ \\
& 90 & $-49^{\dagger}$ & $705^{\dagger}$ \\
& 200 & $-62^{\dagger}$ & $647^{\dagger}$ \\
& 500 & $-72^{\dagger}$ & $638^{\dagger}$ \\
& 10 & 5 & 101 \\
& 30 & 0 & 120 \\
& 90 & $-67^{\dagger}$ & $504^{\dagger}$ \\
& 200 & $-77^{\dagger}$ & $644^{\dagger}$ \\
& 500 & $-93^{\dagger}$ & $699^{\dagger}$
\end{tabular}

Hepatoprotective effects of compounds 1, 2, 13 and $\mathbf{1 4}$ toward in vitro immunological liver injury on primary cultured rat hepatocytes. Significantly different from ref., effective $* p<0.05, * * * p<0.001$. Hepatotoxicity of compounds 1, 2, 13 and 14 in primary cultured rat hepatocytes. Significantly different from ref., toxic $\uparrow p<0.001$. group at $\delta 1.79\left(3 \mathrm{H}, \mathrm{d}, J=6.1 \mathrm{~Hz}, \mathrm{H}_{3}-6\right)$; one olefinic proton at $\delta 5.40(1 \mathrm{H}$, br s); and five anomeric protons at $\delta 5.01(1 \mathrm{H}$, d, $J=6.1 \mathrm{~Hz}), 5.75(1 \mathrm{H}, \mathrm{d}, J=7.9 \mathrm{~Hz}), 5.85(1 \mathrm{H}, \mathrm{d}$, $J=7.3 \mathrm{~Hz}), 6.21(1 \mathrm{H}, J=7.9 \mathrm{~Hz})$, and $6.40(1 \mathrm{H}, \mathrm{br}) . \mathrm{HMBC}$ was observed from the terminal rhamnosyl anomeric proton at $\delta 6.40$ to the inner glucosyl C-2 at $\delta 78.5$; from its inner glucosyl anomeric proton at $\delta 5.01$ to the sapogenol C-3 at $\delta$ 89.8; from the terminal glucosyl anomeric proton at $\delta 5.85$ to the inner glucosyl $\mathrm{C}-2$ at $\delta 78.4$; from another terminal glucosyl anomeric proton at $\delta 5.75$ to the inner glucosyl C-4 at $\delta$ 78.8; and from its inner glucosyl anomeric proton at $\delta$ 6.21 to the sapogenol $\mathrm{C}-28$ at $\delta$ 176.5. Consequently, the structure of BRA-20 (22) was characterized as 3-O- $\alpha$-Lrhamnopyranosyl- $(1 \rightarrow 2)-\beta$-D-glucopyranosyl oleanolic acid 28 - $O$ - $\beta$-D-glucopyranosyl- $(1 \rightarrow 2)$-[ $\beta$-D-glucopyranosyl$(1 \rightarrow 4)]$ - $\beta$-D-glucopyranoside.

BRA-19 (23) was obtained as an amorphous powder having $[\alpha]_{\mathrm{D}}+23.7^{\circ}(\mathrm{MeOH})$. Positive HR-FAB-MS revealed a molecular formula of $\mathrm{C}_{66} \mathrm{H}_{108} \mathrm{O}_{32} \mathrm{Na}$ at $\mathrm{m} / \mathrm{z} \quad 1435.6725$ $[\mathrm{M}+\mathrm{Na}]^{+}$, which corresponds to one mole of hexose more than BRA-20 (22). The ${ }^{13} \mathrm{C}$-NMR spectrum showed the presence of oleanolic acid as a sapogenol, as in BRA-20 (22). HMBC similar to that of $\mathbf{2 2}$ revealed connectivity between the rhamnosyl-( $(1 \rightarrow 2)$-glucosyl- $(1 \rightarrow 2)$-glucosyl- $(1 \rightarrow \mathrm{C}-3$ of sapogenol) and glucosyl-( $1 \rightarrow 2)$-[glucosyl $(1 \rightarrow 4)$-glucosyl$(1 \rightarrow \mathrm{C}-28$ of sapogenol). Therefore, the structure of BRA-19 (23) was characterized as 3-O- $\alpha$-L-rhamnopyranosyl- $(1 \rightarrow 2)$ $\beta$-D-glucopyranosyl- $\beta$-D-glucopyranosyl- $(1 \rightarrow 2)$-oleanolic acid $28-O$ - $\beta$-D-glucopyranosyl- $(1 \rightarrow 2)$-[ $\beta$-D-glucopyranosyl$(1 \rightarrow 4)]$ - $\beta$-D-glucopyranoside.

BRA-14 (24) was obtained as an amorphous powder having $[\alpha]_{\mathrm{D}}+3.2^{\circ}(\mathrm{MeOH})$. Positive HR-FAB-MS revealed a molecular formula of $\mathrm{C}_{54} \mathrm{H}_{88} \mathrm{O}_{23} \mathrm{Na}$ at $\mathrm{m} / \mathrm{z} \quad 1127.5669$ $[\mathrm{M}+\mathrm{Na}]^{+}$. The ${ }^{13} \mathrm{C}-\mathrm{NMR}$ spectrum showed a total of 54 carbon signals, of which 24 were assignable to the sugar moieties rhamnopyranosyl- $(1 \rightarrow 2)$-glucosyl- $(1 \rightarrow$ the $\mathrm{C}-3$ of the sapogenol) and glucosyl-( $1 \rightarrow 4)$-glucosyl- $(1 \rightarrow$ the $\mathrm{C}-28$ of sapogenol). The other 30 carbon signals include one hydroxy carbon at $\delta 74.1$, which was identified as an $\alpha$-hydroxy-bearing carbon at $\mathrm{C}-16$ on oleanolic acid by HMBC. This sapogenol was identified as echinocystic acid. ${ }^{20)}$ Thus, the structure of BRA-14 (24) was characterized as 3-O- $\alpha$-Lrhamnopyranosyl-( $1 \rightarrow 2)-\beta$-D-glucopyranosyl echinocystic acid $\beta$-D-glucopyranosyl-( $1 \rightarrow 2)-\beta$-D-glucopyranoside.

BRA-18 (25) was obtained as an amorphous powder hav-

Table 9. Hepatoprotective Activity of 12-Ene Type Saponins

\begin{tabular}{cccc}
\hline \hline Substances & Dose $(\mu \mathrm{M})$ & Protection (\%) & Toxicity $(\%)$ \\
\hline \multirow{3}{*}{ (Saikosaponin f) } & 10 & -11 & 111 \\
& 30 & 11 & 105 \\
& 90 & 7 & 114 \\
& 200 & 11 & 105 \\
$\mathbf{8}$ (Bupleuroside IX) & 500 & $45^{* * *}$ & 129 \\
& 10 & 2 & 118 \\
& 30 & 8 & 136 \\
& 200 & 10 & $128^{\dagger}$ \\
& 500 & 13 & $124^{\dagger}$ \\
& 27 & $138^{\dagger}$
\end{tabular}

Hepatoprotective effects of compounds $\mathbf{3}$ and $\mathbf{8}$ toward in vitro immunological liver injury on primary cultured rat hepatocytes. Significantly different from ref., effective $* * * p<0.001$. Significantly different from ref., toxic $\uparrow p<0.001$. 
Table 10. Hepatoprotective and Hepatotoxic Activity of 11,13(18)-Diene Type Saponins

\begin{tabular}{|c|c|c|c|}
\hline Substances & Dose $(\mu \mathrm{M})$ & Protection (\%) & Toxicity $(\%)$ \\
\hline \multirow[t]{5}{*}{4 (Saikosaponin $\mathrm{b}_{2}$ ) } & 10 & -74 & 94 \\
\hline & 30 & -59 & 88 \\
\hline & 90 & -50 & 98 \\
\hline & 200 & -28 & 94 \\
\hline & 500 & -108 & 154 \\
\hline \multirow[t]{5}{*}{10 (Saikosaponin $\mathrm{b}_{1}$ ) } & 10 & -10 & 117 \\
\hline & 30 & -7 & 110 \\
\hline & 90 & -23 & 144 \\
\hline & 200 & -3 & 144 \\
\hline & 500 & $-269^{\dagger}$ & 153 \\
\hline \multirow[t]{5}{*}{11 (Saikosaponin h) } & 10 & -43 & ND \\
\hline & 30 & -18 & ND \\
\hline & 90 & -10 & ND \\
\hline & 200 & 8 & ND \\
\hline & 500 & -10 & ND \\
\hline \multirow[t]{5}{*}{15 (Rotundioside E) } & 10 & 22 & ND \\
\hline & 30 & 25 & ND \\
\hline & 90 & -4 & ND \\
\hline & 200 & -19 & ND \\
\hline & 500 & 5 & ND \\
\hline \multirow[t]{5}{*}{16} & 10 & -13 & 124 \\
\hline & 30 & -44 & 123 \\
\hline & 90 & -17 & 103 \\
\hline & 200 & 40 & 118 \\
\hline & 500 & -21 & 135 \\
\hline \multirow[t]{5}{*}{17} & 10 & -31 & ND \\
\hline & 30 & -37 & ND \\
\hline & 90 & -44 & ND \\
\hline & 200 & -16 & ND \\
\hline & 500 & -23 & ND \\
\hline \multirow[t]{5}{*}{18} & 10 & -58 & 103 \\
\hline & 30 & -41 & 91 \\
\hline & 90 & -28 & 100 \\
\hline & 200 & -10 & 97 \\
\hline & 500 & 41 & 93 \\
\hline \multirow[t]{5}{*}{19} & 10 & 0 & ND \\
\hline & 30 & 14 & ND \\
\hline & 90 & 9 & ND \\
\hline & 200 & 21 & ND \\
\hline & 500 & 32 & ND \\
\hline \multirow[t]{5}{*}{20} & 10 & -43 & ND \\
\hline & 30 & -28 & ND \\
\hline & 90 & -21 & ND \\
\hline & 200 & -12 & ND \\
\hline & 500 & 43 & ND \\
\hline \multirow[t]{5}{*}{21} & 10 & -38 & ND \\
\hline & 30 & -48 & ND \\
\hline & 90 & -31 & ND \\
\hline & 200 & -3 & ND \\
\hline & 500 & 27 & ND \\
\hline
\end{tabular}

Hepatoprotective effects of compounds 4, 10, 11 and 15-21 toward in vitro immunological liver injury on primary cultured rat hepatocytes. Significantly different from ref. Hepatotoxicity of compounds $4,10,11$ and $15-21$ in primary cultured rat hepatocytes. Significantly different from ref., toxic $\uparrow p<0.001$.

ing $[\alpha]_{\mathrm{D}}+2.7^{\circ}(\mathrm{MeOH})$. Positive HR-FAB-MS revealed a molecular formula of $\mathrm{C}_{60} \mathrm{H}_{98} \mathrm{O}_{28} \mathrm{Na}$ at $\mathrm{m} / \mathrm{z} \quad 1289.6140$ $[\mathrm{M}+\mathrm{Na}]^{+}$. The ${ }^{13} \mathrm{C}-\mathrm{NMR}$ spectrum showed 60 carbon signals consisting of echinocystic acid and the same sugar moieties as in BRA-20 (22). Therefore, the structure of BRA-18 (25) was characterized as 3-O- $\alpha$-L-rhamnopyranosyl- $(1 \rightarrow 2)$ $\beta$-D-glucopyranosyl echinocystic acid $\beta$-D-glucopyranosyl$(1 \rightarrow 2)$-[ $\beta$-D-glucopyranosyl- $(1 \rightarrow 4)]-\beta$-D-glucopyranoside.

We isolated a total of 25 oleanene saponins from the aerial parts of B. falcatum and B. rotundifolium and the seeds of $B$.
Table 11. Hepatoprotective and Hepatotoxic Activity of 28-Acid Type Saponins

\begin{tabular}{cccc}
\hline \hline \multirow{2}{*}{ Substances } & Dose $(\mu \mathrm{M})$ & Protection $(\%)$ & Toxicity $(\%)$ \\
\hline \multirow{2}{*}{24} & 10 & 15 & 99 \\
& 30 & 33 & 98 \\
90 & $79 * * *$ & 83 \\
& 200 & $55^{* * *}$ & 89 \\
500 & $54 * *$ & 114 \\
& 10 & -6 & 133 \\
& 30 & 6 & 125 \\
& 90 & 4 & 95 \\
& 200 & 0 & 112 \\
& 500 & 22 & 107
\end{tabular}

Hepatoprotective effects of compounds $\mathbf{2 4}$ and $\mathbf{2 5}$ toward in vitro immunological liver injury on primary cultured rat hepatocytes. Significantly different from ref., effective $* * p<0.01, * * * p<0.001$. Hepatotoxicity of compounds 24 and 25 in primary cultured rat hepatocytes.

falcatum. They are divided into four groups: the 13,28-epoxy type, the 12-ene type, the 9(11),12-diene type, and the 28acid type, according to their structural characteristics.

Next, some compounds obtained from them were investigated for their hepatoprotective activity and hepatotoxicity.

At the 13,28-epoxy type saponins, 2 (saikosaponin c) had weak hepatoprotectivity at $500 \mu \mathrm{M}$, but 1 (saikosaponin a) showed hapatotoxity from $30 \mu \mathrm{M}$. It was suggested that methyl group of the 23 position of $\mathbf{1}$ influenced activity. ${ }^{21}$ ) But compound $\mathbf{3}$ of which an epoxy ring cleaved at C-13,28 of $\mathbf{1}$ has hepatoprotective activity at $500 \mu \mathrm{M}$. Ursane type (13, 14) showed hepatotoxity from middle concentration. It was guessed that conformation of 29 and 30 position methyl group in ursane skelton damaged against cell membranes. On the other hands, 11,13(18)-diene type saponins did not express hepatoprotective activity and $\mathbf{4}$ and $\mathbf{1 0}$ showed the toxicity from middle concentration among them. The 28-acid type saponin $(\mathbf{2 4}, \mathbf{2 5})$ which has a glucosyl carboxy group showed hepatoprotective action. This result accorded with our previous paper. ${ }^{22)}$

\section{Experimental}

General Procedure Optical rotations were measured with a JASCO P$1020(l=0.5)$ automatic digital polarimeter. FAB-MS were obtained with a glycerol matrix in the positive ion mode using a JEOL JMS-DX300 and a JMS-DX $303 \mathrm{HF}$ spectrometer. The ${ }^{1} \mathrm{H}$ - and ${ }^{13} \mathrm{C}-\mathrm{NMR}$ spectra were measured in pyridine- $d_{5}$ with JOEL $\alpha-500$ spectrometer, and chemical shifts are given on a $\delta(\mathrm{ppm})$ scale with tetramethylsilane (TMS) as the internal standard. Column chromatographies were carried out on a Diaion HP-20 (Mitsubishi Chemical Ind.), and silica gel 60 (230 - 400 mesh, Merck). TLC was performed on silica gel plates (Kieselgel $60 \mathrm{~F}_{254}$, Merck) and $\mathrm{PR} \mathrm{C}_{18}$ silica gel plates (Merck). The spots on TLC were visualized by UV light $(254 / 366 \mathrm{~nm})$ and sprayed with $10 \% \mathrm{H}_{2} \mathrm{SO}_{4}$, followed by heating.

Extraction and Isolation of Respective Plants The aerial parts $(2.6 \mathrm{~kg})$ of Bupleurum falcatum L. cultivated at Kikka-cho, Kumamoto prefecture, was cutted and refluxed with $\mathrm{MeOH}$ for $10 \mathrm{~h}$. Evaporation of $\mathrm{MeOH}$ gave the extractive $(262.2 \mathrm{~g})$, which was partitioned between $n$-hexane and $80 \%$ $\mathrm{MeOH}$, and the methanolic layer was evaporated to give the residue. It was then chromatographed on Diaion HP-20 eluted with firstly water and gradiently mixed with $\mathrm{MeOH}$ to provide five fractions, fr. 1 (132.0 g), fr. 2 (48.7 g), fr.3 (25.6 g), fr. 4 (14.6g), and fr. 5 (12.4g). The fr. 4 eluted by $80 \% \mathrm{MeOH}$ was subjected Sephadex LH-20 with $\mathrm{MeOH}$ to afford flavonoid and saponin faractions, from the latter of which was chromatographed on Chromatorex ODS with $60-100 \% \mathrm{MeOH}$ to give BFA-5 (3, $108 \mathrm{mg})$, BFA$6(4,264 \mathrm{mg})$, BFA-8 (1, $45 \mathrm{mg})$, BFA-11 (21 mg), BFA-13 (110 mg), BFA$17(7,21 \mathrm{mg})$, BFA-20 (6, $20 \mathrm{mg})$, BFA-22 $(2,72 \mathrm{mg})$. The fr. 5 eluate by $100 \% \mathrm{MeOH}$ was also subjected to silica gel chromatography with $\mathrm{CHCl}_{3}-$ $\mathrm{MeOH}=15: 1-\mathrm{CHCl}_{3}-\mathrm{MeOH}-$ water $=9: 1: 0.1$ to give $\mathrm{BFA}-25(5,17 \mathrm{mg})$. 
The seeds $(1.2 \mathrm{~kg})$ of Bupleurum falcatum L. used for cultivating at Kikka-cho were smashed and extracted with $\mathrm{MeOH}$ for $1 \mathrm{~h}$, and usual workup gave the extractive ( $84.7 \mathrm{~g}$ ), which was subjected to Diaion HP-20 with water- $\mathrm{MeOH}$, gradiently, finally recovered with acetone) to provide seven fractions. The fr. $5(23.2 \mathrm{~g})$ eluted by $80 \% \mathrm{MeOH}$ was chromatographed on silica gel with $\mathrm{CHCl}_{3}-\mathrm{MeOH}$-water $=9: 1: 0.1-7: 3: 0.5$ to afford fr. $5-1-$ fr. 5-8. The fr. 5-1 (3.0 g) was further purified by silicagel with $\mathrm{CHCl}_{3}-$ $\mathrm{MeOH}=50: 1$ and prepaartive TLC with $\mathrm{CHCl}_{3}-\mathrm{MeOH}=15: 1$ to give BFS-8 $(9,8 \mathrm{mg})$. The fr. 5-4 $(1.5 \mathrm{~g})$ was chromatographed by HPLC to give BAS-9 $(\mathbf{1}, 13 \mathrm{mg})$, BAS-10 $(\mathbf{1 2}, 31 \mathrm{mg})$, and BAS-11 (10, $34 \mathrm{mg})$. From fr. 5-5 (14.6 g), fr. 5-6 (1.3 g), and fr. 5-8 (1.4 g), the respective BAS-13 (4, $28 \mathrm{mg})$, BAS-14 $(\mathbf{1 1}, 15 \mathrm{mg})$, and BAS-1 $(2,30 \mathrm{mg})$ were obtained by using HPLC with $80 \% \mathrm{MeOH}$. The fr. $6(9.5 \mathrm{~g})$ eluted by $\mathrm{MeOH}$ was chromatographed on silica gel with $\mathrm{CHCl}_{3}-\mathrm{MeOH}$-water $=8: 2: 0.2$ to give BAS-16 (8,15 mg).

The aerial parts $(1.4 \mathrm{~kg})$ of Bupleurum rotundifolium L. cultivated at Oyano, Kumamoto prefecture, was cutted and refluxed with $\mathrm{MeOH}$ for $7 \mathrm{~h}$. Evaporation of $\mathrm{MeOH}$ gave the extractive $(152.1 \mathrm{~g})$, which was defatted with $n$-hexane. The insoluble residue was passed through Diaion HP-20 eluted with water- $\mathrm{MeOH}$, gradiently, to give five fractions. A part ( $2.3 \mathrm{~g})$ of the fr. $4(6.6 \mathrm{~g})$ eluted by $80 \% \mathrm{MeOH}$ was various chromatographed on silica gel with $\mathrm{CHCl}_{3}-\mathrm{MeOH}$-water $=8: 2: 0.2$, Chromatorex ODS with $50-100 \%$ $\mathrm{MeOH}$, gradiently, and HPLC with $60 \% \mathrm{MeOH}$ to give BRA-11 (19, $36 \mathrm{mg})$, BRA-14 (24, $69 \mathrm{mg})$, BRA-16 (20, $12 \mathrm{mg})$, BRA-17 (21, $11 \mathrm{mg})$, BRA-22 (18, $22 \mathrm{mg})$, BRA-18 $(\mathbf{2 5}, 38 \mathrm{mg})$, BRA-19 (23, $6 \mathrm{mg})$, and BRA$20(22,5 \mathrm{mg})$. From a part $(6.5 \mathrm{~g})$ of the fr. $5(22.3 \mathrm{~g})$ eluted by $\mathrm{MeOH}$, BRA-2 (15, $19 \mathrm{mg})$, BRA-3 (13, $19 \mathrm{mg})$, BRA-4 (16, $35 \mathrm{mg})$, BRA-5 (14, $60 \mathrm{mg})$, BRA-7 (17, $14 \mathrm{mg})$ by using silica gel and ODS column chromatographies with $\mathrm{CHCl}_{3}-\mathrm{MeOH}-$ water $=8: 2: 0.2-7: 3: 0.5$, and ODS with $60-100 \% \mathrm{MeOH}$, gradiently.

Identification of Component Monosaccharide of the Glycosides A glycoside $(5 \mathrm{mg})$ was dissolved in $1 \mathrm{~N} \mathrm{HCl}-\mathrm{MeOH}(0.5 \mathrm{ml})$ and heated at $90^{\circ} \mathrm{C}$ for $1 \mathrm{~h}$. The acidic solution was neutralized with an ion exchanger resin (Amberlite IR-410) and concentrated in vacuo. The residue was trimethylsilyalted and checked by gas-liquid chromatography (GLC). Authentic sugar samples were treated in the same manner and $t_{\mathrm{R}}$ values were compared with those of the tetramethylsilyl derivatives of the metahnolysate of the glycoside.

The absolute configurations of the component monosaccharides were determined according to the method reported by Hara et al. Thus, a glycoside $(5 \mathrm{mg})$ was hydrolyzed with $1 \mathrm{~N} \mathrm{HCl}$. After neutralization with Amberliet IR410 , the free sugars in the hydrolysate were converted into the thiazolidine derivatives and checked by GLC after trimethylsilylation. Authentic sugar samples were treated in the same manner and an unknown sugar was identified by comparison of its $t_{\mathrm{R}}$ value with those of the authentic sugar deriavtives.

Animals Male Wistar rats (6 weeks old, body weight $150-160 \mathrm{~g}$ ) and a male New Zealand white rabbit (body weight $3 \mathrm{~kg}$ ) were used.

Preparation of Primary Cultured Rat Hepatocytes Liver cells were isolated to a procedure developed by Berry and Friend. ${ }^{23)}$

Preparation of Antiserum against Rat Hepatocytes The antiserum was prepared according to the method of Shiki et al. ${ }^{24)}$ An antibody to rat hepatocytes was raised in rabbits, first by injection of $1 \times 10^{8}$ cells, followed by four injections of $5 \times 10^{7}$ cells over a period of 4 weeks. The antiserum to the rat hepatocytes was prepared by the method of Harboe and Ingild. ${ }^{25)}$

Determination of Hepatoprotective Activity of Compounds toward in Vitro Immunological Liver Injury One day after the isolated rat hepatocytes were plated, the cultured cells were exposed to the above medium $(300 \mu \mathrm{l})$ containing the antiserum against rat plasma membranes $(80 \mu \mathrm{l} / \mathrm{ml})$ and a dimethyl sulfoxide (DMSO) solution $(4 \mu \mathrm{l})$ of the test samples [final concentration 0 (reference; Ref.), 10, 30, 90, 200, $500 \mu \mathrm{M}]$. Forty min after the antiserum was administered, the medium was withdrawn for determination of alanine aminotransferase (ALT). Control is the value of hepatocytes which were not administered the antiserum. The control was hepatocytes not treated with antiserum. The control value $\mathbf{1 , 2 , 1 3}$ and $\mathbf{1 4}$ was $6.48 \pm 1.4$ (IU/1). The value of 1 at 0 (Ref.), 10, 30, 90, 200, $500 \mu \mathrm{M}$ were $27.50 \pm 4.1$, $24.75 \pm 2.9,29.25 \pm 3.0,34.50 \pm 2.2,33.75 \pm 1.0,33.25 \pm 1.7$ (IU/1), respectively. The value of 2 at 0 (ref.), 10, 30, 90, 200, $500 \mu \mathrm{M}$ were $27.50 \pm 0.6$, $24.75 \pm 1.7,27.00 \pm 0.8,24.75 \pm 1.0,23.25 \pm 1.0,18.75 \pm 0.5$ (IU/1), respectively. The value of $\mathbf{1 3}$ at 0 (Ref.), $10,30,90,200,500 \mu \mathrm{M}$ were $26.25 \pm 4.9$, $26.50 \pm, 36.50 \pm 10.2,36.25 \pm 3.1,39.00 \pm 5.1,41.00 \pm 1.5$ (IU/1), respectively. The value of $\mathbf{1 4}$ at 0 (Ref.), 10, 30, 90, 200, $500 \mu \mathrm{M}$ were $26.00 \pm 4.9$, $25.00 \pm 4.5,26.00 \pm 1.0,39.00 \pm 3.1,41.00 \pm 5.1,44.25 \pm 1.5$ (IU/1), respectively. The control value $\mathbf{3}$ and $\mathbf{8}$ was 17.54 \pm 1.4 (IU/1). The value of $\mathbf{3}$ at 0
(Ref.), 10, 30, 90, 200, $500 \mu \mathrm{M}$ were $31.50 \pm 0.6,33.00 \pm 0.5,30.00 \pm 1.4$, $30.50 \pm 1.0,30.00 \pm, 25.25 \pm 0.5$ (IU/1), respectively. The value of 8 at 0 (Ref.), 10, 30, 90, 200, $500 \mu \mathrm{M}$ were $33.25 \pm 2.1,33.00 \pm 2.1,32.00 \pm 0.8$, $31.75 \pm 1.5,31.25 \pm 2.0,29.00 \pm 1.2$ (IU/1), respectively. The control value 4 , $10,11,15,16,17,18,19,20$ and 21 was $9.17 \pm 1.4$ (IU/1). The value of 4 at 0 (Ref.), 10, 30, 90, 200, $500 \mu \mathrm{M}$ were $17.25 \pm 1.4,23.25 \pm 2.9,22.00 \pm 1.7$, $21.25 \pm 1.8,19.50 \pm 3.3,26.00 \pm 1.0$ (IU/1), respectively. The value of $\mathbf{1 0}$ at 0 (Ref.), 10, 30, 90, 200, $500 \mu \mathrm{M}$ were $26.25 \pm 2.7,28.00 \pm 15,27.50 \pm 1.7$, $30.25 \pm 3.3,26.75 \pm 2.1,72.25 \pm 3.0$ (IU/1), respectively. The value of 11 at 0 (Ref.), 10, 30, 90, 200, 500 $\mu \mathrm{M}$ were $19.00 \pm 2.4,23.25 \pm 1.3,20.75 \pm 2.6$, $20.00 \pm 3.8,18.25 \pm 1.5,20.00 \pm 2.8$ (IU/1), respectively. The value of $\mathbf{1 5}$ at 0 (Ref.), 10, 30, 90, 200, $500 \mu \mathrm{M}$ were $33.25 \pm 3.0,28.00 \pm 6.1,27.25 \pm 1.9$, $34.25 \pm 5.3,37.75 \pm 4.1,32.00 \pm 1.8(\mathrm{IU} / 1)$, respectively. The value of $\mathbf{1 6}$ at 0 (Ref.), 10, 30, 90, 200, $500 \mu \mathrm{M}$ were $21.00 \pm 2.2,22.50 \pm 1.0,26.25 \pm 4.2$, $23.00 \pm 1.4,16.25 \pm 0.5,23.50 \pm 1.3$ (IU/1), respectively. The value of $\mathbf{1 7}$ at 0 (Ref.), 10, 30, 90, 200, $500 \mu \mathrm{M}$ were $18.75 \pm 2.2,21.75 \pm 1.7,22.25 \pm 3.2$, $23.00 \pm 1.8,20.25 \pm 0.9,21.00 \pm 1.8$ (IU/1), respectively. The value of $\mathbf{1 8}$ at 0 (Ref.), 10, 30, 90, 200, $500 \mu \mathrm{M}$ were $19.00 \pm 1.8,24.75 \pm 4.3,23.00 \pm 3.4$, $21.75 \pm 3.3,20.00 \pm 1.2,15.00 \pm 1.8(\mathrm{IU} / \mathrm{l})$, respectively. The value of $\mathbf{1 9}$ at 0 (Ref.), 10, 30, 90, 200, $500 \mu \mathrm{M}$ were $23.2 \pm 1.0,23.25 \pm 0.5,21.25 \pm 2.1$, $22.00 \pm 1.2,20.25 \pm 1.3,18.75 \pm 3.1(\mathrm{IU} / 1)$, respectively. The value of $\mathbf{2 0}$ at 0 (Ref.), 10, 30, 90, 200, $500 \mu \mathrm{M}$ were $19.75 \pm 3.0,24.25 \pm 2.5,22.75 \pm 2.2$, $22.00 \pm 1.4,21.00 \pm 3.0,15.25 \pm 3.0$ (IU/1), respectively. The value of 21 at 0 (Ref.), 10, 30, 90, 200, $500 \mu \mathrm{M}$ were $16.50 \pm 3.0,19.25 \pm 1.7,20.00 \pm 2.7$, $18.75 \pm 1.3,16.75 \pm 1.7,14.50 \pm 1.7$ (IU/1), respectively. The control value 24 and $\mathbf{2 5}$ was $17.54 \pm 1.4$ (IU/1). The value of $\mathbf{2 4}$ at 0 (Ref.), 10, 30, 90, 200, $500 \mu \mathrm{M}$ were $38.75 \pm 2.5,35.50 \pm 1.3,31.75 \pm 1.7,22.00 \pm 3.2,27.00 \pm 2.2$, $27.25 \pm 5.0$ (IU/1), respectively. The value of $\mathbf{2 5}$ at 0 (Ref.), 10, 30, 90, 200, $500 \mu \mathrm{M}$ were $30.25 \pm 1.5,29.50 \pm 0.6,29.50 \pm 1.3,29.75 \pm 1.3,30.25 \pm 2.2$, $27.50 \pm 1.3$ (IU/1), respectively. Reference (ref.) value treated with the antiserum and not treated with the tested sample. The percent of protection is calculated as $\{1-($ substance - control $) /($ ref. - control $)\} \times 100$. The percent of protection of glycyrrhizin (positive control) was $32 \%$ at $500 \mu \mathrm{M}$.

Determination of Hepatotoxicity of Saponins toward Hepatocytes (without Anitiserum) In the same way as above, the cultured cells were exposed to the above-prepared medium $(300 \mu$ l) containing the DMSO solution $(4 \mu \mathrm{l})$ of the test samples [final concentration 0 (Ref.), 10, 30, 90, 200, $500 \mu \mathrm{M}]$. Forty minute after the test samples were administered, the medium was withdrawn for determination of ALT. The value of $\mathbf{1}$ at 0 (Ref.), 10, 30, $90,200,500 \mu \mathrm{M}$ were $24.80 \pm 2.1,23.80 \pm 5.5,78.50 \pm 5.0,122.00 \pm 3.7$, $112.80 \pm 1.9,96.75 \pm 3.9$ (IU/1), respectively. The value of $\mathbf{1 3}$ at 0 (Ref.), 10, $30,90,200,500 \mu \mathrm{M}$ were $19.25 \pm 5.7,21.25 \pm 4.8,116.5 \pm 4.4,135.80 \pm 13.7$, $124.80 \pm 4.8,122.80 \pm 5.3$ (IU/1), respectively. The value of $\mathbf{1 4}$ at 0 (Ref.), 10, $30,90,200,500 \mu \mathrm{M}$ were $20.50 \pm 3.7,20.75 \pm 2.2,24.50 \pm 0.6,103.30 \pm 13.3$, $132.00 \pm 7.0,143.30 \pm 12.0$ (IU/1), respectively. The value of 3 at 0 (Ref.), 10, $30,90,200,500 \mu \mathrm{M}$ were $14.00 \pm 2.0,15.50 \pm 0.6,14.75 \pm 2.4,16.00 \pm 0.9$, $14.75 \pm 1.5,18.00 \pm 1.6$ (IU/1), respectively. The value of 8 at 0 (Ref.), 10, 30 , $90,200,500 \mu \mathrm{M}$ were $12.50 \pm 2.1,14.75 \pm 1.0,17.00 \pm 2.8,13.00 \pm 1.7$, $15.50 \pm 1.3,17.25 \pm 2.1$ (IU/1), respectively. The value of 4 at 0 (Ref.), 10, 30, $90,200,500 \mu \mathrm{M}$ were $17.25 \pm 1.4,23.25 \pm 2.9,22.00 \pm 1.7,21.25 \pm 1.8$, $19.50 \pm 3.3,26.00 \pm 1.0$ (IU/1), respectively. The value of $\mathbf{1 0}$ at 0 (Ref.), 10, $30,90,200,500 \mu \mathrm{M}$ were $26.25 \pm 2.7,28.00 \pm 1.5,27.50 \pm 1.7,30.25 \pm 3.3$, $26.75 \pm 3.0,27.25 \pm 1.7$ (IU/1), respectively. The value of $\mathbf{1 6}$ at 0 (Ref.), 10, $30,90,200,500 \mu \mathrm{M}$ were $21.00 \pm 2.2,22.50 \pm 1.0,26.25 \pm 4.2,23.00 \pm 1.4$, $16.25 \pm 0.5,23.5 \pm 1.7$ (IU/1), respectively. The value of $\mathbf{1 8}$ at 0 (Ref.), 10, 30, $90,200,500 \mu \mathrm{M}$ were $19.00 \pm 1.8,24.75 \pm 4.3,23.00 \pm 3.4,21.75 \pm 3.3$, $20.00 \pm 1.2,15.00 \pm 0.8$ (IU/1), respectively. The percent of cytotoxicity is calculated as (sample/reference) $\times 100$. Reference is the value of hepatocytes which were not treated with the tested samples. The value of $\mathbf{2 4}$ at 0 (Ref.), $10,30,90,200, \quad 500 \mu \mathrm{M}$ were $20.75 \pm 2.5,20.50 \pm 1.3,20.25 \pm 1.7$, $17.25 \pm 3.2,18.50 \pm 2.2,23.75 \pm 5.0$ (IU/1), respectively. The value of 25 at 0 (Ref.), 10, 30, 90, 200, $500 \mu \mathrm{M}$ were $19.00 \pm 1.5,25.25 \pm 0.6,23.75 \pm 1.3$, $18.00 \pm 1.3,21.25 \pm 2.2,20.25 \pm 1.3$ (IU/1), respectively. The percent of cytotoxicity is calculated as (sample/reference) $\times 100$. Reference is the value of hepatocytes which were not treated with the tested samples. The percent of cytotoxicity is calculated as (sample/reference) $\times 100$. Reference is the value of hepatocytes which were not treated with the tested samples.

Instrument and Assay Method The activities of ALT were assayed by autoanalyzer COBAS MIRA (Roche) using commercial kits based on the ALT assay method. ${ }^{26)}$

Statistical Analysis The data are shown as the mean \pm S.D. $(n=4)$. After analysis of variances, Sheffe's test was employed to determine the significance of differences between reference and experimental samples. 


\section{References}

1) Shibata S., Kitagawa I., Fujimoto H., Chem. Pharm. Bull., 14, 1023 1033 (1966).

2) Shimaoka A., Seo S., Minato H., J. C. S., Perkin 1, 1975, 2043-2048 (1975).

3) Tori K., Yoshimura Y., Seo S., Sakurai K., Ishii H., Tetrahedron Lett., 46, 4163-4166 (1976).

4) Shimizu K., Amagaya S., Ogihara Y., Chem. Pharm. Bull., 33, 33493355 (1985).

5) Nose M., Amagaya S., Takeda T., Ogihara Y., Chem. Pharm. Bull., 37, 1293-1296 (1989).

6) Nose M., Amagaya S., Ogihara Y., Chem. Pharm. Bull., 37, 27362740 (1989).

7) Ebita N., Nakajima K., Taguchi H., Mitsuhashi H., Chem. Pharm. Bull., 38, 1432-1434 (1990).

8) Yoshikawa M., Murakami T., Hirano K., Inadzuki M., Ninomiya K., Matsuda H., Tetrahedron Lett., 38, 7395-7398 (1989).

9) Matsuda H., Murakami T., Ninomiya K., Inadzuki M., Yoshikawa M., Bioorg. Med. Chem. Lett., 7, 2193-2198 (1997).

10) Ono M., Yoshida A., Ito Y., Nohara T., Phytochemistry, 51, 819-823 (1999).

11) Fujioka T., Yoshida K., Fujii H., Nagao T., Okabe H., Mihashi K., Chem. Pharm. Bull., 51, 365-372 (2003).

12) Fujioka T., Yoshida K., Shibao H., Nagao T., Yoshida M., Matsunaga K., Takata J., Karube Y., Iwase Y., Okabe H., Mihashi K., Mihashi K., Chem. Pharm. Bull., 54, 1694-1704 (2006).
13) Ishi H., Nakamura M., Chem. Pharm. Bull., 28, 2367-2383 (1980).

14) Kircher H. W., Phytochemistry, 16, 1078-1080 (1977).

15) Kobayashi Y., Takeda T., Ogihara Y., Chem. Pharm. Bull., 29, 2222 2229 (1981).

16) Navarro P., Ginder R. M., Life Sci., 68, 1199-1206 (2001).

17) Barrero A. F., Haïdour A., Sedqui A., Mansour A. I., RodríguezGarcia I., Löpez A., Muñoz-Dorado M., Phytochemistry, 54, 741745 (2000).

18) Sachez-Contreras S., Diaz-Lanza A. M., Phytochemistry, 54, 783789 (2000).

19) Kobaayshi Y., Ogihara Y., Chem. Pharm. Bull., 29, 2230-2236 (1981).

20) Akai E., Takeda T., Kobayashi Y., Chen Y., Ogihara Y., Chem. Pharm. Bull., 33, 4685-4690(1985).

21) Kinjo J., Udayama M., Okawa M., Nohara T., Biol. Pharm. Bull., 22 203-206 (1999).

22) Kinjo J., Okawa M., Udayama M., Sohno Y., Hirakawa T., Shii Y., Nohara T., Chem. Pharm. Bull., 47, 290-292 (1999).

23) Berry M. N., Friend D. S., J. Cell Biol., 43, 506-520 (1969).

24) Shiki Y., Shirai K., Saito Y., Yoshida S., Wakashin M., Kumagai A., Wakan-Yaku Gakkaishi, 1, 11-14 (1984).

25) Harboe N., Ingild A., Scand. J. Immunol., 2 (Suppl. 1), 161-164 (1973).

26) Heerspink W., Hafkenscheid J. C., Siepel H., van der Ven-Jongekrÿg J., Dijt C. C., Enzyme, 25, 333-341 (1980). 